\title{
Dust in active galactic nuclei
}

\section{Mid-infrared T-ReCS/Gemini spectra using the new RedCan pipeline ${ }^{\star}$}

\author{
O. González-Martín ${ }^{1,2}$, J. M. Rodríguez-Espinosa ${ }^{1,2}$, T. Díaz-Santos ${ }^{3}$, C. Packham ${ }^{4}$, A. Alonso-Herrero ${ }^{5, \star \star}$, \\ P. Esquej ${ }^{5,6,7}$, C. Ramos Almeida ${ }^{1,2}$, R. Mason $^{8}$, and C. Telesco ${ }^{9}$ \\ 1 Instituto de Astrofísica de Canarias (IAC), C/Vía Láctea, s/n, 38205 La Laguna, Spain \\ e-mail: omairagm@iac.es \\ 2 Departamento de Astrofísica, Universidad de La Laguna (ULL), 38205 La Laguna, Spain \\ 3 Spitzer Science Center, California Institute of Technology, 1200 East California Boulevard, Pasadena, CA 91125, USA \\ ${ }^{4}$ Dept. of Physics \& Astronomy, University of Texas San Antonio, San Antonio, TX. 78249, USA \\ 5 Instituto de Física de Cantabria, CSIC-Universidad de Cantabria, 39005 Santander, Spain \\ 6 Departamento de Física Moderna, Universidad de Cantabria, Avda. de Los Castros s/n, 39005 Santander, Spain \\ 7 Centro de Astrobiología, INTA-CSIC, 28850 Madrid, Spain \\ ${ }^{8}$ Gemini Observatory, Northern Operations Center, 670 N. A'ohoku Place, Hilo, HI 96720, USA \\ 9 Department of Astronomy, University of Florida, Gainesville, Florida 32611, USA
}

Received 13 September 2012 / Accepted 21 December 2012

\begin{abstract}
Context. The unified model of active galactic nuclei (AGN) claims that the properties of AGN depend on the viewing angle of the observer with respect to a toroidal distribution of dust surrounding the nucleus. Both the mid-infrared (MIR) attenuation and continuum luminosity are expected to be related to dust associated with the torus. Therefore, isolating the nuclear component is essential for studying the MIR emission of AGN.

Aims. This work is aimed at studying the MIR emission of AGN with the highest spatial resolution available to date, isolating its contribution from extended emission. We would like to address three fundamental questions: (1) how important is the AGN contribution to the MIR spectrum; (2) where dust attenuation arises; and (3) how does spatial resolution affect these issues.

Methods. We compiled all the T-ReCS spectra (Gemini Observatory) available in the $N$-band for 22 AGN: 5 Type-1 and 17 Type-2 AGN. The high angular resolution of the T-ReCS spectra allowed us to probe physical regions of $57 \mathrm{pc}$ (median). We used a novel pipeline called RedCan capable of producing flux- and wavelength-calibrated spectra for the CanariCam (GTC) and T-ReCS (Gemini) instruments. We measured the fine-structure [S IV] at $10.5 \mu \mathrm{m}$ and the PAH at $11.3 \mu \mathrm{m}$ line strengths, together with the silicate absorption/emission features. We also compiled Spitzer/IRS spectra to understand how spatial resolution influences the results. We complemented our sample with the results of 19 VISIR/VLT spectra (Paranal Observatory) and 20 nearby, highly obscured AGN $\left(N_{\mathrm{H}}>1.5 \times 10^{24} \mathrm{~cm}^{-2}\right)$ Spitzer spectra.

Results. The $11.3 \mu \mathrm{m}$ PAH feature is only clearly detected in the nuclear spectra of two AGN, while it is more common in the Spitzer data. For those two objects, the AGN emission in NGC 7130 accounts for more than $80 \%$ of the MIR continuum at $12 \mu \mathrm{m}$, while in the case of NGC 1808 the AGN does not dominate the MIR emission. This is confirmed by the correlation between the MIR and X-ray continuum luminosities. The [S IV] emission line at $10.5 \mu \mathrm{m}$, which is believed to originate in the narrow line region, is detected in most AGN. We have found an enhancement of the optical depth at $9.7 \mu \mathrm{m}\left(\tau_{9.7}\right)$ in the high-angular resolution data for higher values of $N_{\mathrm{H}}$. Clumpy torus models reproduce the observed values only if the host-galaxy properties are taken into account.
\end{abstract}

Key words. accretion, accretion disks - galaxies: active - galaxies: Seyfert - infrared: galaxies - techniques: imaging spectroscopy

\section{Introduction}

The radiation from active galactic nuclei (AGN) is caused by accretion onto a super-massive black hole (SMBH). The optical spectra of AGN have been classified into two main classes according to the existence (Type-1) or not (Type-2) of broad permitted lines $\left(F W H M>2000 \mathrm{~km} \mathrm{~s}^{-1}\right)$. The so-called unified model proposes that both types of AGN are essentially the same objects viewed at different angles (Antonucci 1993; Urry \& Padovani 1995). An optically-thick dusty torus surrounding

\footnotetext{
* Appendix $\mathrm{A}$ is available in electronic form at http://www . aanda.org

$\star \star$ Augusto Gonzalez Linares Senior Research Fellow.
}

the central source would be responsible for blocking the region where these broad emission lines are produced (the so-called broad line region, BLR). Therefore, Type-2 AGN are essentially Type-1 AGN blocked by the dusty torus along the line of sight (LOS) to the observer. More elaborated models suggest that the same observational constraints can also be explained with a torus made of discrete dense molecular clouds following a given radial distribution (Hönig et al. 2006; Schartmann et al. 2008; Elitzur 2008; Nenkova et al. 2008a,b; Hönig \& Kishimoto 2010b).

Mid-infrared (MIR) spectroscopy is a powerful tool for examining the nature of AGN, as well as the contribution of star formation activity. Weedman et al. (2005) find a variety of 
spectral features displayed in the MIR spectra of eight classical AGN with Spitzer data (see also Rodriguez-Espinosa et al. 1987, 1997). Large samples of AGN observed with Spitzer have been studied in detail to quantify the properties of AGN at MIR (e.g. Sturm et al. 2006; Deo et al. 2007; Hao et al. 2007).

Among the most noteworthy results found at MIR wavelengths, Wu et al. (2009) find that the silicate features at $9.7 \mu \mathrm{m}$ and $18 \mu \mathrm{m}$ in Type- 1 Seyfert galaxies are rather weak, while Type-2 Seyfert galaxies are likely to correspond to strong silicate absorptions. Furthermore, the deepest silicate absorption features show the highest values for the hydrogen column density $\left(N_{\mathrm{H}}\right)$ in X-rays (Shi et al. 2006). The silicate emission feature in Type-1 AGN is much weaker than expected from smooth dusty tori. The clumpiness of the dusty torus explains the overall suppression of this feature better (Nenkova et al. 2002, 2008a). However, clumpy models do not predict strong silicate absorption features because illuminated and shadowed clouds contribute to the observed spectrum. In fact, the silicate feature depth typically seen in some Compton-thick AGN $\left(N_{\mathrm{H}}>1.5 \times 10^{24} \mathrm{~cm}^{-2}\right)$ observed with Spitzer is similar to what is observed in Type-1 AGN (Goulding et al. 2012). However, the nature of these silicate feature is not clear since the host-galaxy could be another contributor to the dust attenuation seen in the MIR (see also Alonso-Herrero et al. 2011; Deo et al. 2007).

Another prominent feature noticeable in the MIR is the polycyclic aromatic hydrocarbon (PAH) emission. This is usually prominent in starburst galaxies, with a good correlation between the strength of the PAHs and the IR luminosity in starbursts (Brandl et al. 2006). This correlation appears, however, to be weak or absent in AGN (Weedman et al. 2005). PAHs form in the photodissociation regions (PDRs) in star-forming clouds, but these PDRs can easily be destroyed by the intense radiation field from the AGN, even if it is a vigorous starburst (Genzel et al. 1998; Laurent et al. 2000). Goulding et al. (2012), using low resolution Spitzer spectra, found that Compton-thick AGN show strong PAH features, suggesting that the PDRs can survive the AGN radiation field if properly shielded. Diamond-Stanic \& Rieke (2012) found a relation between the accretion rate of AGN and the SFR derived from PAHs. They suggest that the gas fueling the SMBH is also used for the star formation.

However, the relatively low spatial resolution of Spitzer/IRS spectra $(\sim 3$ arcsec) includes a non-negligible contribution of the host galaxy and/or of circumnuclear star formation. A good isolation of the torus emission is therefore needed to understand the pure AGN contribution to the MIR spectrum. The best isolation possible to date can be obtained using IR interferometry (e.g. Tristram et al. 2007, 2009). However, this can only be done for a few bright AGN. As for the rest of AGN, the medium- to large-size ground-based telescopes offer an opportunity to better isolate the contribution of the AGN within a few tens of parsecs. Some individual works have been presented showing the importance of spatial resolution to isolate the AGN from the host galaxy (e.g. NGC 1068, NGC 1365, NGC 3281, and the Circinus galaxy; Mason et al. 2006; Alonso-Herrero et al. 2012; Sales et al. 2011; Roche et al. 2006, respectively). A large sample was presented by Hönig et al. (2010a), including 19 AGN observed with the VLT/VISIR MIR spectrograph with spatial resolution better than $\sim 100 \mathrm{pc}$. They found moderately deep silicate absorption features. However, this sample is free of objects with high inclination angles or dust lanes, and include only three Compton-thick AGN. The MIR luminosity seems to be quite isotropic, correlating with other AGN luminosity tracers (see also Ramos Almeida et al. 2007; Levenson et al. 2009; Gandhi et al. 2009; Mason et al. 2012).
In this paper, we compile all the AGN observed by T-ReCS at the Gemini Observatory. The sample comprises 22 AGN, including nine Compton-thick sources. Our main aims are (1) to characterize the AGN pure emission at MIR frequencies; (2) to understand the nature of the attenuation seen at MIR frequencies as traced by the optical depth at $9.7 \mu \mathrm{m}$; (3) to compare T-ReCS and Spitzer spectra to understand the limitations introduced by the lower spatial resolution in Spitzer spectra.

For the data processing and analysis we used a newly developed pipeline called RedCan ${ }^{1}$. This pipeline is able to produce flux-calibrated images and 1D spectra for both the T-ReCS (Telesco et al. 1998; De Buizer \& Fisher 2005) and the CanariCam $^{2}$ (Telesco et al. 2003) instruments without any user interaction. This pipeline has been produced to better exploit of the CanariCam data, and it can be freely accessed (see Sect. 3).

\section{Sample selection}

We have searched for all the T-ReCS (Gemini South Observatory) AGN spectra available in the Gemini public archive $^{3}$ until June 2012. The sample comprises 22 AGN observed as part of about ten observational projects. All of them are observed in the $N$-band. Since T-ReCS is not expected to be available after 2013A, this sample includes most of the AGN ever to be observed with this instrument ${ }^{4}$.

Table 1 shows the main observational details of the sample. Seventeen objects are Type- 2 and five are Type-1 AGN. The slit width used for the spectroscopy results in a spatial resolution between $\sim 7-236$ pc for all the objects except for 3C 445 (406 pc), which is by far the most distant object in the sample. This is the largest sample of high spatial resolution spectra ever put together, especially when including the 19 AGN (VISIR/VLT) reported by Hönig et al. (2010a). Our sample includes nine Compton-thick AGN (see $N_{\mathrm{H}}$ in Table 1), making it complementary to the AGN sample in Hönig et al. (2010a) (where only 3 Compton-thick AGN are included); however, our sample is unbiased though not complete in any sense since the observations come from several different proposals with differing goals. It is worth noticing that this sample appears to contain a high fraction of sources with large silicate absorption features (see Sect. 6).

\section{Data reduction: the CanariCam data reduction pipeline (RedCan)}

Data reduction pipelines are an important aspect of modern astronomy. Indeed large telescope instruments are becoming fairly complex, so that the prompt availability of science data is crucial for securing fast scientific turnout from today's large and expensive facilities. Unfortunately, this is not always the case, even in successful facilities. The aim of our group is to produce a new and advanced pipeline for MIR imaging and spectroscopic data. In the forthcoming years, large amounts of GTC/CanariCam data will be acquired by our group. This includes data from both guaranteed CanariCam time $(\sim 100 \mathrm{~h}, \mathrm{PI} \mathrm{C}$. Telesco) and the ESO/GTC proposal led by A. Alonso-Herrero $(\sim 180 \mathrm{~h}$, ESO/GTC 182.B.2005). It is therefore important to have a data

\footnotetext{
1 The CanariCam data reduction pipeline.

2 CanariCam is a mid-infrared $(7.5-25 \mu \mathrm{m})$ imager with spectroscopic, coronagraphic, and polarimetric capabilities, which is mounted at one of the Nasmyth foci of the GTC telescope at El Roque observatory.

3 http://www3.cadc-ccda.hia-iha.nrc-cnrc.gc.ca/

cadcbin/gsa/wdbi.cgi/gsa/gsa_science/form

4 Except for three objects that are not public yet.
} 
Table 1. Sample properties.

\begin{tabular}{|c|c|c|c|c|c|c|c|c|c|}
\hline $\begin{array}{l}\text { Num } \\
\text { (1) }\end{array}$ & $\begin{array}{l}\text { Name } \\
(2)\end{array}$ & $\begin{array}{l}D(\mathrm{Mpc}) \\
(3)\end{array}$ & $\begin{array}{c}\text { Slit width }(\operatorname{arcsec}) / \operatorname{Size}(p c) \\
(4)\end{array}$ & $\begin{array}{c}\text { Type } \\
(5)\end{array}$ & $\begin{array}{l}\text { Optical features } \\
\text { (6) }\end{array}$ & $\begin{array}{c}\log (N \mathrm{H}) \\
(7)\end{array}$ & $\begin{array}{c}\log (L(2-10 \mathrm{keV})) \\
(8)\end{array}$ & $\begin{array}{l}\text { X-ray Ref. } \\
\text { (9) }\end{array}$ & $\begin{array}{l}b / a \\
(10)\end{array}$ \\
\hline 1 & NGC 1365 & 17.9 & $0.35 / 30$ & S1.5 & & 23.6 & 42.5 & $(1,11)$ & 0.5 \\
\hline 2 & NGC 1386 & 16.2 & $0.31 / 24$ & S2 & $\mathrm{E}$ & $>25.0$ & 42.6 & $(2,3)$ & 0.4 \\
\hline 3 & NGC 1808 & 11.5 & $0.35 / 19$ & $\mathrm{~S} 2$ & & 22.9 & 40.4 & $(3,12)$ & 0.6 \\
\hline 4 & NGC 3081 & 32.5 & $0.65 / 102$ & $\mathrm{~S} 2$ & & 23.9 & 42.5 & (2) & 0.8 \\
\hline 5 & NGC 3094 & 44.7 & $0.35 / 76$ & S2 & & & & & 0.7 \\
\hline 6 & NGC 3281 & 45.7 & $0.35 / 77$ & S2 & $\mathrm{D}, \mathrm{E}$ & 24.3 & 43.4 & $(1,12)$ & 0.4 \\
\hline 7 & NGC 4418 & 31.1 & $0.35 / 53$ & S2 & E & $>25.0$ & 41.2 & (4) & 0.4 \\
\hline 8 & NGC 4945 & 4.0 & $0.65 / 13$ & S2 & $\mathrm{D}, \mathrm{E}, \mathrm{M}$ & 24.7 & 42.3 & $(5,12)$ & 0.4 \\
\hline 9 & Centaurus A & 3.7 & $0.65 / 12$ & S2 & $\mathrm{D}, \mathrm{E}$ & 23.7 & 41.9 & $(1,13)$ & 0.4 \\
\hline 10 & NGC 5135 & 58.6 & $0.70 / 199$ & S2 & & $>25.0$ & 43.1 & (2) & 0.7 \\
\hline 11 & Circinus & 4.2 & $0.35 / 7$ & S2 & $\mathrm{M}$ & 24.6 & 42.6 & $(6,2)$ & 0.5 \\
\hline 12 & NGC 5506 & 28.8 & $0.35 / 49$ & S1.9 & $\mathrm{D}, \mathrm{E}$ & 22.5 & 43.0 & $(1,3)$ & 0.2 \\
\hline 13 & NGC 5643 & 16.9 & $0.35 / 29$ & S2 & & 23.8 & 42.6 & $(7,12)$ & 0.9 \\
\hline 14 & NGC 5728 & 29.1 & $0.35 / 49$ & S1.9 & & 23.6 & 43.3 & (6) & 0.6 \\
\hline 15 & IC $4518 \mathrm{~W}$ & 69.6 & $0.70 / 236$ & S2 & $\mathrm{E}, \mathrm{M}$ & 24.3 & 42.6 & $(8,13)$ & 0.1 \\
\hline 16 & ESO 103-G35 & 56.9 & $0.35 / 96$ & S2 & E & 23.2 & 43.4 & $(1,12)$ & 0.4 \\
\hline 17 & IC 5063 & 48.6 & $0.65 / 153$ & S2 & D & 23.3 & 43.3 & $(1,12)$ & 0.7 \\
\hline 18 & NGC 7130 & 69.2 & $0.70 / 235$ & L2 & M & $>25.0$ & 43.1 & $(10,2)$ & 0.9 \\
\hline 19 & NGC 7172 & 33.9 & $0.35 / 57$ & S2 & D & 22.9 & 42.8 & $(1,3)$ & 0.6 \\
\hline 20 & $3 \mathrm{C} 445$ & 239.3 & $0.35 / 406$ & $\mathrm{~S} 1.5$ & & 23.5 & 43.9 & (3) & 0.9 \\
\hline 21 & NGC 7479 & 33.8 & $0.35 / 57$ & L1.9 & $\mathrm{D}, \mathrm{M}$ & 24.3 & 42.0 & (3) & 0.8 \\
\hline 22 & NGC 7582 & 20.6 & $0.70 / 70$ & S2 & $\mathrm{D}, \mathrm{E}$ & 23.0 & 42.6 & (2) & 0.4 \\
\hline
\end{tabular}

Notes. The size of the region observed by T-ReCS is computed using the slit width. Optical features: (D) Dust features across the nuclear region; (E) Edge-on galaxies; (M) Merger.

References. The references for the X-ray results are: (1) Tueller et al. (2008); (2) Marinucci et al. (2012); (3) Brightman \& Nandra (2011); (4) Maiolino et al. (2003); (5) Itoh et al. (2008); (6) Goulding et al. (2012); (7) Guainazzi et al. (2004); (8) Pereira-Santaella et al. (2011); (9) Moran et al. (1996); (10) Mullaney et al. (2011); (11) Risaliti et al. (2009); (12) Comastri (2004); and (13) de La Rosa et al. (2011).

reduction pipeline that facilitates the use of the data as soon as possible after being acquired at the telescope. This is the purpose of RedCan, the Canaricam data reduction pipeline we have developed. RedCan is a well-tested reduction and calibration procedure to ensure reliable data products from CanariCam.

Thanks to the similarities between the T-ReCS and CanariCam instruments, RedCan can be used for data from both instruments in both imaging and spectroscopy modes $(Q$ and $N$ bands in medium resolution).

RedCan has been written by combining the Gemini IRAF package $^{5}$ ) within PyRAF, together with ad hoc IDL routines developed for this purpose. CSHELL language is used for the main routine of the pipeline to connect IDL and Python routines $^{6}$. Figure 1 shows a chart flow of the reduction steps done by RedCan to produce the final reduced data. RedCan is based on the procedure described by Díaz-Santos et al. (2010, see also Díaz-Santos et al. 2008), and the main input file is an ASCII file with a list of observations. A full description of the pipeline procedure is given below.

\subsection{Identification of files}

A full data set from a target observation consists of (1) telluric standard observations; (2) target observation; and (3) acquisitions of both standard and target images. Standards and targets

\footnotetext{
5 The released version of the Gemini IRAF package is an external package layered upon IRAF and is available to users and other interested parties (http://www.gemini.edu/sciops/ data-and-results/processing-software).

6 The RedCan package can be retrieved here: https://www. dropbox.com/sh/w4z8buo2ewrhhvj/Kk1f2RYVe8/RedCan.tar . gz. Note that the pipeline is of free access, although it will not be supported. A manual is also available that includes documentation on the software requirements and installation procedures.
}

can be in imaging and/or spectroscopic modes. Moreover, the acquisition images in the case of spectroscopic data can be either imaging or imaging throughout the slit.

RedCan uses keywords in the fit headers to identify the type of each observing block, and they are used accordingly throughout the pipeline. In particular the keyword OBSCLASS are used together with the instrumental configuration keywords (i.e. SLIT, GRATING, FILTER1, FILTER 2, etc.) and observational keywords (i.e. ONTIME, RA, DEC, UT, DATE) to identify the following information:

- Acquisition image associated to a target/standard. RedCan sorts the files according to the observed time and selects those immediately before the target/standard as acquisition images. Acquisition images of the objects through the slit are used to compute slit losses for the spectroscopic mode (see Sect. 3.8). These images are also flux-calibrated during the process so they can be used for science purposes.

- Standard star image/spectrum associated to a target. RedCan selects within the same date of observation the standard image/spectrum observed with the same configuration used for the target (i.e. same filter and grating configuration with the same slit size). If more than one observation of standard stars fulfil this criterion, the one closer in time is chosen. This association is used to flux-calibrate both images and spectra (see Sects. 3.4 and 3.8).

\subsection{Flat fielding}

The RedCan pipeline allows subtracting a master flat computed as an average of the observations of the sky for each chop integration. This master flat is computed using the tasks TPREPARE and MISTACK within the midir/IRAF package, while the flat subtraction is implemented in IDL. Díaz-Santos et al. (2008) 


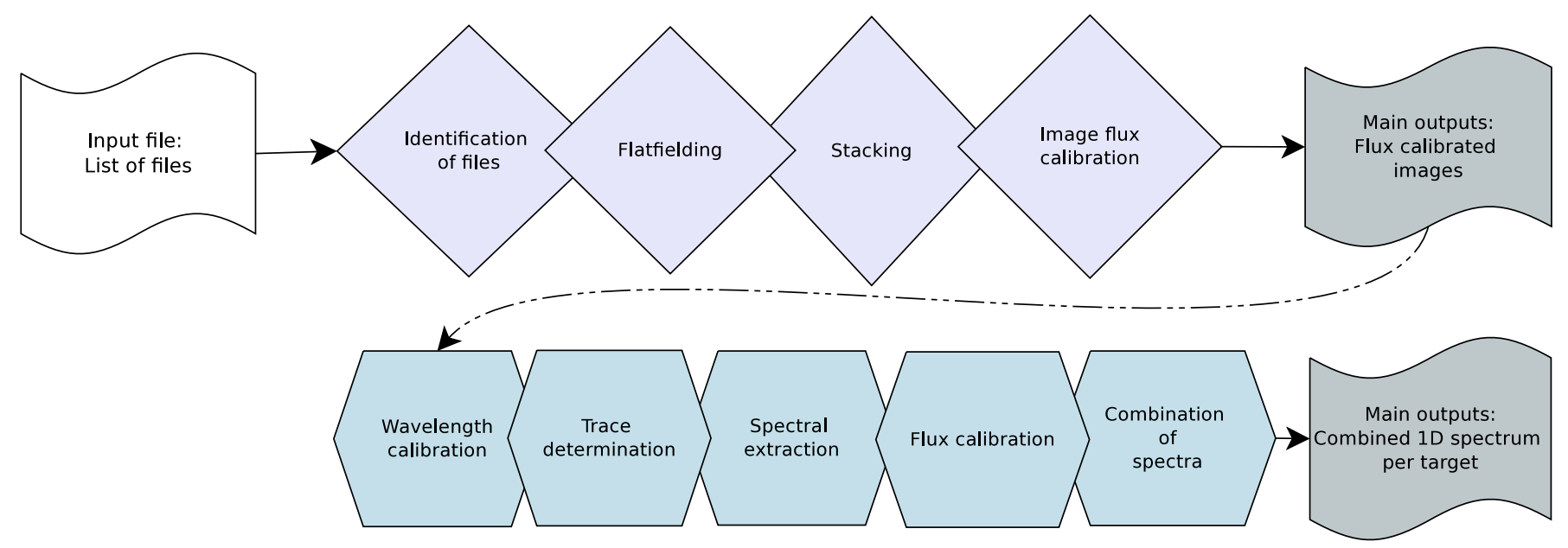

Fig. 1. Flowchart of the RedCan procedure. Diamonds are the steps in the imaging procedure, while polygons indicate the steps in the spectroscopic procedure. The empty flag is the input level and the dark grey flags indicate the output levels. The dashed arrow is set to point out that RedCan continues from imaging to spectroscopic procedures only when spectra are included in the initial dataset.

conclude that flat-fielding can improve the quality of the final images. However, the flat-fielding procedure introduces noise into the final image (see e.g. the Gemini web page ${ }^{7}$ ). We have detected that using flat-fielding in the spectroscopic mode can lead to follow a wrong trace of the spectra due to including patterns at the borders of the array. This step is included in RedCan as an option for the user, although the default is set to skip it. We skipped this option in the data presented in this paper.

\subsection{Stacking}

Accurate cancellation of the sky and telescope background is most readily achieved through the technique of chopping and nodding. The sky is very bright and highly variable at MIR frequencies when observed from the Earth. To get rid of the sky, the chop technique frequency observes a nearby sky position (off-source chop) moving the secondary mirror of the telescope to subtract from the target position (on-source chop). However, this chopping technique leaves a background resulting from the different paths followed by the light depending on whether the telescope is pointing to the on-source or the off-source positions. The nodding technique places the source at the nod positions, moving the actual telescope axes to remove this residual. Thus, the chop-nod technique allows subtraction of the sky background, as well as of the background from the telescope itself. This technique also removes the array $1 / \mathrm{f}$ noise. The data are then stored automatically in save sets. Prior to stacking, RedCan automatically rejects chop-nod pairs of images affected by noise patterns or glitches due to instrumental or background problems during the observations. This rejection is done by default excluding any chop-nod pair above or below $5 \sigma$ of the mean value of the subtracted chop-nod pair of images (task TBACKGROUND within Gemini/midir). However, this can be executed manually, and the threshold level can be changed if desired. Both the onsource and the off-source chop images are averaged together (task MIPREPARE) and each nod pair combined (task MISTACK). The resulting image shows certain types of pattern noise as horizontal or vertical stripes. Those that are constant along the image are removed with the task MICLEAN within the gemini/midir package. Finally each image is divided by the frame time so the final image is in units of "adu/s".

\footnotetext{
7 http://www.gemini.edu/?q=node/10139\#flat
}

The final product is a single data cube per observation with three planes: on source, off source, and on-off source (i.e. background subtracted) images. This procedure is followed for every image (i.e. acquisitions, standards, and targets). The second plane is needed for wavelength calibration in the spectroscopic mode (see Sect. 3.5).

\subsection{Imaging flux calibration}

Images resulting from the stacking process described above are then flux calibrated. This flux calibration is performed for both acquisition and target images using associated telluric standards stars.

RedCan contains the catalogue of synthetic flux-calibrated spectra of standards stars published by Cohen et al. (1999). An identification of the standard star is done using the coordinates given in the header of the observation. When the standard star is not part of the catalogue of Cohen, a reference standard star is used, and a warning is shown in the screen and recorded in the output tables and log of the reduction process. Then, the synthetic spectrum of the star is convolved with the telescope throughput and filter bandpass. Aperture photometry of the standard star is performed, and a new keyword called FCALSTD is placed in the standard star header, which provides the ratio between the number of counts per second and the real flux of the star. Then aperture photometry techniques are applied to the target images, and a table with the final fluxes for the set of images is recorded. The aperture radii of the standard star and target are fixed to a radius of 2.7 and 0.9 arcsec respectively. These radii include more than $95 \%$ of the flux at the diffraction limit in both T-ReCS $\left(F W H M \sim 0.35-0.37 \operatorname{arcsec}^{8}\right)$ and CanariCam data $\left(F W H M \sim 0.26-0.42 \operatorname{arcsec}^{9}\right)$ and above $90 \%$ with good seeing conditions (optical seeing below 1 arcsec). The background is extracted using annuli with internal and external radii of $[4.5,5.3]$ and $[1.8,2.25]$ arcsec, respectively. Finally, fluxcalibrated images are also produced for target and standard stars using the conversion factor stored in FCALSTD.

This method is flux accurate to $10 \%$ (Díaz-Santos et al. 2008). However, we note that this aperture photometry is accurate enough for point-like sources, while a more sophisticated

\footnotetext{
8 http://www.gemini.edu/?q=node/10582\#trecspsf

9 http://www.gtc.iac.es/en/pages/instrumentation/ canaricam/data-commissioning.php\#Image_Quality
} 
methodology is needed for extended sources (see Sect. 4.1). RedCan finishes here if the input list of files does not include any spectroscopic data. It continues to the next steps otherwise.

\subsection{Wavelength calibration}

RedCan uses sky emission features in the reference stacked spectra to wavelength-calibrate all the spectra (standard stars and targets). First, the sky spectrum is stacked along the spatial axis. The pipeline looks for the local maxima in this stacked spectrum as local peaks within the ten closest pixels. A Gaussian profile is fitted to the 11 pixels centred in the local maxima to accurately determine the position of the centre. This method is fairly sensitive to broad features such as those from the MIR sky.

The next step is to identify these local maxima with known features. For that purpose the pipeline contains a set of wavelength-calibrated sky templates for the $N$ and $Q$ band and for T-ReCS and CanariCam. These observational templates are obtained from the best weather conditions data from the Gemini archive for T-ReCS and commissioning data for CanariCam data. After selecting the associated template according to the grating and instrument, a grid of offsets and distortions is applied to it. The best offset and distortion for the sky spectrum is chosen by computing the minimum $\chi_{r}^{2}$. Using the wavelength calibration of the templates, RedCan identifies as many features as possible in the sky-spectrum. A re-identification of these lines along the spatial axis is made every ten pixels. A linear fit is then applied in the wavelength direction, and third and fourth order fits are applied in the spatial direction for T-ReCS and CanariCam data respectively. The final calibration is stored in a $2 \mathrm{D}$ wavelengthcalibration array.

The $N$-band spectral resolution is $R=100$ for T-ReCS and $R=175$ for CanariCam. The accuracy of the wavelength calibration is always much better than the spectral resolution of the instruments.

\subsection{Trace determination}

Before extracting the spectra the traces of standard stars and targets are computed. The pipeline stacks the spectrum along the wavelength axis every ten pixels and determines the centre and width of the trace by fitting it to a MOFFAT profile (the mpfitpeak $^{10}$ function of IDL). The resulting widths of the trace in the spatial direction are fitted to a polynomial. A first and second order degree polynomials are tested against the $N$-band spectra to include the possibility that the data might not be diffractionlimited at bluer wavelengths. For the width and centre of the trace in the $Q$-band, a linear fit is used for both T-ReCS and CanariCam. The centre and width of the trace for each observation are saved in its header with the keywords TRCTRA, TRCTRB, TRFWHMA, TRFWHMB, and TRFWHMC.

\subsection{Spectral extraction}

RedCan extracts the spectrum by using the 2-D final spectrum of the target and standard star and the wavelength calibration matrix (see Sect. 3.5). For the centre of the extraction region, the pipeline uses the centre of the trace for each object recorded in the header (see Sect. 3.6). For the width of the extraction region

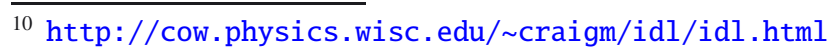

two considerations can be made: (1) extended or (2) point-like sources $^{11}$.

For point-like sources the width in the spatial direction as a function of wavelength is set to vary as the trace of the associated standard star. The user can specify the number of times the width of the standard to be used. To extract the unresolved component, this number should be set to one. For extended sources the width does not vary with wavelength, and the user can specify the number of pixels to be extracted. Additionally, a number of offsets (in pixels) from the centre of the target (i.e. centre of the trace) can be specified to extract one or several regions out of the centre of the target. The pipeline can extract either several widths for an offset or several offsets for a given width.

For our AGN sample we have selected the point-like source option for unresolved sources using the width of the standard star to isolate the nuclear component from the extended emission.

\subsection{Spectral flux calibration and combination of spectra}

Each spectrum is flux-calibrated using the ratio between the observed associated standard and its corresponding flux-calibrated template given by Cohen et al. (1999). For the extraction of the standard a radius of four times the sigma of the trace of the standard is used to ensure the collection of the whole flux of the standard.

For extended sources, the final flux-calibrated spectrum is obtained by dividing the spectrum (in counts) by this ratio. For point-like sources, to obtain a well flux-calibrated spectrum, losses due to the width of the slit (slit-losses) and due to the aperture used (aperture-losses) are taken into account. The slit losses are automatically computed using the acquisition images for the associated standard star. Two images must be present within the observations, before and after including the slit in the optical system. The pipeline computes aperture photometry in these two images and puts the ratio of the counts of the observations without slit divided by the counts using the slit in the header, adding the new keyword SLITCORR in the observation corresponding to the spectrum of the star. When the user selects the option pointlike the final spectrum is multiplied by this value to obtain the final flux-calibrated spectrum. Also, slit losses are wavelengthdependent for point-like sources because the angular size of the PSF in a diffraction limited observation increases linearly with wavelength. Therefore the loss ratio calculated using the acquisition images, with and without slit, is only a first-order correction, since it is based on the averaged flux of the source as measured through the filter used for each acquisition and thus is a constant value. While this is not important for point-source extractions, it is a source of uncertainty for extended sources. When observing unresolved sources, both the standard and the target are affected by the same wavelength-dependent slit loss, which is automatically accounted for when the spectrum of the target is divided by that of the standard. On the other hand, extended sources are assumed as not having any loss due to either the slit or aperture. Therefore the standard star introduces an uncertainty into the shape of the extracted spectrum of the target even when the standard is corrected by the "constant" loss ratio.

For the aperture losses the standard star is extracted with the same width as the target. These aperture losses depend on the wavelength, and they are computed as the ratio between the standard spectrum (with 4 times the standard trace) and the spectrum extracted with the same trace as the target. The errors are estimated as the sum of the statistical error (squared root of the

${ }_{11}$ These two options can be selected by the user. 
Table 2. Observational details of the T-ReCS spectra.

\begin{tabular}{|c|c|c|c|c|c|c|}
\hline Name & ObsID & Expt. & Date & PA & STD* & $\begin{array}{c}F W H M \\
(8.7 \mu \mathrm{m}) \\
(7)\end{array}$ \\
\hline NGC 1365 & 0061 & 624 & 090829 & 15 & 26967 & 0.14 \\
\hline NGC 1386 & 0210,12 & $2 \times 606$ & 060929 & 0 & 23319 & 0.29 \\
\hline NGC 1808 & 0076 & 981 & 090829 & 45 & 26967 & 0.20 \\
\hline \multirow[t]{2}{*}{ NGC 3081} & 0094 & 606 & 060125 & 0 & 81797 & 0.20 \\
\hline & 0113 & 606 & 060304 & 350 & 93813 & 0.29 \\
\hline NGC 3094 & 0075,76 & $2 \times 309$ & 040506 & 0 & 81797 & 1.10 \\
\hline \multirow[t]{2}{*}{ NGC 3281} & 0120 & 981 & 090328 & 315 & HR3438* & 0.17 \\
\hline & 0033,35 & $2 \times 981$ & 090406 & 315 & 146791 & 0.20 \\
\hline \multirow[t]{3}{*}{ NGC 4418} & 0041 & 309 & 040507 & 40 & 128620 & 0.25 \\
\hline & 0046,47 & $2 \times 309$ & 040508 & 40 & 128620 & 0.13 \\
\hline & $0025-28$ & $4 \times 327$ & 080628 & 30 & 99998 & 0.30 \\
\hline NGC 4945 & 0055,56 & $2 \times 303$ & 060417 & 45 & 110458 & 0.26 \\
\hline Centaurus A & 0198 & 517 & 040131 & 0 & 123139 & 0.22 \\
\hline \multirow[t]{2}{*}{ NGC 5135} & 0098 & 633 & 060306 & 30 & 101666 & 0.19 \\
\hline & 0106,07 & $2 \times 633$ & 060310 & 30 & 101666 & 0.16 \\
\hline \multirow[t]{2}{*}{ CIRCINUS } & 0084,85 & $2 \times 309$ & 040506 & 100 & 81797 & 1.10 \\
\hline & 0095-97 & $3 \times 309$ & 040506 & 10 & 81797 & 1.10 \\
\hline NGC 5506 & 0107,08 & $2 \times 309$ & 040506 & 0 & 81797 & 1.10 \\
\hline \multirow[t]{3}{*}{ NGC 5643} & $0057-60$ & $4 \times 303$ & 070605 & 80 & 123139 & 0.21 \\
\hline & $0057-59$ & $3 \times 327$ & 080615 & 80 & 123139 & 0.50 \\
\hline & $0060-62$ & $6 \times 327$ & 080615 & 80 & 139127 & 0.40 \\
\hline NGC 5728 & 0046,47 & $2 \times 921$ & 090406 & 215 & 146791 & 0.18 \\
\hline IC 4518W & $0081-83$ & $3 \times 633$ & 060417 & 5 & 123123 & 0.22 \\
\hline \multirow[t]{2}{*}{ ESO 103-G35 } & 0065-67 & $3 \times 318$ & 080616 & 30 & 156277 & 0.24 \\
\hline & $0068-70$ & $3 \times 318$ & 080616 & 30 & 171759 & 0.51 \\
\hline \multirow[t]{5}{*}{ IC 5063} & 0078 & 606 & 050521 & 303 & 81797 & 0.20 \\
\hline & 0046 & 606 & 050707 & 303 & 81797 & 0.20 \\
\hline & 0047 & 303 & 050707 & 303 & 81797 & 0.20 \\
\hline & 0048 & 302 & 050707 & 303 & 81797 & 0.20 \\
\hline & 0092 & 302 & 050709 & 303 & 81797 & 0.20 \\
\hline \multirow[t]{7}{*}{ NGC 7130} & 0061 & 633 & 050918 & 348 & 190056 & 0.30 \\
\hline & 0062 & 633 & 050918 & 348 & 219784 & 0.35 \\
\hline & 0111-113 & $3 \times 633$ & 060704 & 348 & 190056 & 0.14 \\
\hline & 0057 & 633 & 060829 & 348 & 190056 & 0.24 \\
\hline & 0107,08 & $2 \times 633$ & 060916 & 348 & 190056 & 0.19 \\
\hline & 0097,98 & $2 \times 633$ & 060925 & 348 & 190056 & 0.25 \\
\hline & 0099 & 633 & 060925 & 348 & 219784 & 0.29 \\
\hline \multirow[t]{4}{*}{ NGC 7172} & $0120-22$ & $3 \times 309$ & 040506 & 60 & 81797 & 1.10 \\
\hline & 0077-80 & $4 \times 327$ & 080615 & 90 & 200914 & 0.29 \\
\hline & 0041,42 & $2 \times 318$ & 080625 & 90 & 200914 & 0.34 \\
\hline & 0063-66 & $4 \times 327$ & 080627 & 90 & 200914 & 0.25 \\
\hline $3 \mathrm{C} 445$ & 0098,99 & $2 \times 921$ & 100606 & 300 & 206445 & 0.24 \\
\hline \multirow[t]{2}{*}{ NGC 7479} & $0055-57$ & $3 \times 327$ & 080629 & 10 & 206445 & 0.41 \\
\hline & 0058-60 & $3 \times 327$ & 080629 & 10 & 1255 & 0.23 \\
\hline NGC 7582 & 0174,75 & $2 \times 606$ & 050718 & 0 & BS8556* & 0.20 \\
\hline
\end{tabular}

Notes. ${ }^{(*)}$ STD: Standard star used to perform flux-calibration. All the standard stars are named with their HD sequence number except those marked with a star. The ObsID (Col. 2) includes the last 4 digits of the name of the observing file. The first 8 digits are the date of the observation (i.e. "S"+ObsID+"S20"+YYMMDD).

number of counts) plus a $15 \%$ systematic errors due to the flux calibration procedure (see Díaz-Santos et al. 2008).

The observations are grouped together into targets using the keyword OBJNAME in the headers. All the flux- and wavelengthcalibrated spectra of the same target are combined into one. This final spectrum is given at rest frame by using the redshift obtained from $\mathrm{NED}^{12}$. For this the name of the target is used. When the object is not found in NED (either because it is not extragalactic or the name is not correctly spelled in the header), a redshift equal to zero is used. The applied redshift is stored in an ascii file.

Table 2 shows the observational details for our sample. In particular, this includes information on the standard star used to

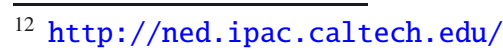
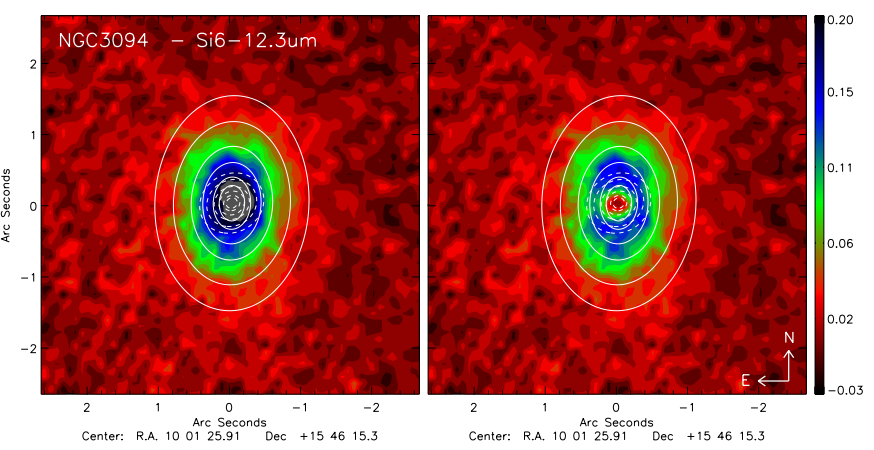

Fig. 2. Si6 image at $12.3 \mu \mathrm{m}$ of NGC 3094 before (left panel) and after (right panel) the PSF subtraction. In the latter, one can clearly see the nuclear emission in red. The dashed contour is the best MOFFAT fit for the standard star. The colour scale is in units of Jy.

flux-calibrate each observation and the number of observations combined to produce the final spectrum of each target.

\section{Data analysis}

\subsection{MIR images}

RedCan produces flux-calibrated images (see Sect. 3.4) using the associated standard star images and their theoretical spectra given by Cohen et al. (1999). Figure 2 (left) shows the resulting flux-calibrated image for NGC 3094. Most of the $N$-band images are acquisition images associated to the target. For that reason most of the sources have imaging in the $N$-band (except Centaurus A, NGC 5506, and NGC 5728). $Q$-band filter images are available for five objects, while the narrow band filters $\mathrm{Si} 2$ $(8.8 \mu \mathrm{m})$ and $\mathrm{Si} 6(12.3 \mu \mathrm{m})$ are available for nine and three sources, respectively. All sources have an image in at least one of the filters.

Our aim is to study the central region morphology of the sample in the MIR and isolate the AGN contribution. We have used the closest (in time) standard star observed with the same filter as representative of the PSF of a point-like source. This star is fitted to a 2D MOFFAT profile (using the "mpfit2dpeak" IDL function). We have scaled this PSF function to the peak of the nuclear source and subtracted the nuclear component. This method would overestimate the nuclear flux of the source if extended. However, it is not our aim to measure fluxes (they are estimated with the spectra) and this approximation is robust at determining the number of extended sources.

The right hand panel in Fig. 2 shows the residual after the subtraction of the PSF (dashed contours) for NGC 3094. We consider a source as extended if the image after the PSF subtraction shows an integrated flux higher than three times the standard deviation of the background. Column 2 in Table 3 indicates whether the sources are extended $(\mathrm{E})$ or point-like $(\mathrm{P})$.

In general, for the sources in our sample, the central morphology is extended. Only six AGN show pointlike nuclear emission, namely NGC 1365, Centaurus A, NGC 5728, IC 4518W, ESO 103-G35, and NGC 7479. However, Alonso-Herrero et al. (2012) found an extended morphology for NGC 1365 using $N$-band images with T-ReCS. Their proprietary data have a higher $\mathrm{S} / \mathrm{N}$ since our image is only the acquisition image for the spectrum. Thus, the $\mathrm{S} / \mathrm{N}$ influences the identification of the extended emission in NGC 1365. In the cases where the source is not extended the nuclear emission comes from less than 7-236 pc, depending on the distance to the galaxies. The sources observed using several filters show 
Table 3. Main results.

\begin{tabular}{|c|c|c|c|c|c|c|c|c|c|c|c|c|}
\hline \multirow[t]{3}{*}{ Name } & \multirow[t]{3}{*}{$\mathrm{E} / \mathrm{P}$} & \multicolumn{3}{|c|}{$\tau_{9.7}$} & \multicolumn{2}{|c|}{$f_{v=12 \mu \mathrm{m}}(\mathrm{mJy})$} & \multicolumn{2}{|c|}{$\log \left(v L_{v=12 \mu \mathrm{m}}(\mathrm{erg} / \mathrm{s})\right)$} & \multicolumn{2}{|c|}{$E W(\mathrm{PAH} 11.3 \mu \mathrm{m})$} & \multicolumn{2}{|c|}{$E W([\mathrm{SIV}])$} \\
\hline & & T-ReCS & Spit & zer & T-ReCS & Spitzer & T-ReCS & Spitzer & T-ReCS & Spitzer & T-ReCS & Spitzer \\
\hline & & & (linear) & (PAHFIT) & & & & & & & & \\
\hline NGC 1365 & $P$ & $-0.03 \pm 0.03$ & $0.20 \pm 0.01$ & $0.00 \pm 0.01$ & $373.1 \pm 9.8$ & $752.9 \pm 2.0$ & 42.71 & 43.02 & $<31.3$ & 113.6 & $<8.9$ & 13.1 \\
\hline NGC 1386 & $\mathrm{E}$ & $0.73 \pm 0.16$ & $0.68 \pm 0.01$ & $0.53 \pm 0.01$ & $291.2 \pm 44.3$ & $498.0 \pm 6.0$ & 42.52 & 42.75 & $<41.8^{* *}$ & 47.6 & 56.3 & 32.4 \\
\hline NGC 1808 & $\mathrm{E}$ & $0.53 \pm 0.03$ & $1.17 \pm 0.06$ & $0.67 \pm 0.01$ & $293.7 \pm 7.8$ & $683.8 \pm 18.7$ & 42.23 & 42.59 & 183.1 & 520.8 & $<27.5$ & 14.6 \\
\hline NGC 3081 & $\mathrm{E}$ & $0.14 \pm 0.10$ & $0.24 \pm 0.03$ & $0.00 \pm 0.01$ & $177.7 \pm 19.4$ & $255.0 \pm 2.5$ & 42.91 & 43.06 & $<12.1$ & 34.1 & 36.3 & 60.8 \\
\hline NGC 3094 & $\mathrm{E}$ & $3.04 \pm 0.19$ & & & $734.6 \pm 11.3$ & & 43.80 & & $<8.1$ & $\ldots$ & $<88.8$ & \\
\hline NGC 3281 & $\mathrm{E}$ & $1.21 \pm 0.11$ & $1.15 \pm 0.07$ & $1.29 \pm 0.05$ & $813.3 \pm 94.1$ & $691.8 \pm 81.2$ & 43.86 & 43.79 & $<7.7$ & & $<5.8^{* *}$ & 14.6 \\
\hline NGC 4418 & E & $3.78 \pm 0.27$ & $3.59 \pm 0.06$ & $2.60 \pm 0.05$ & $735.0 \pm 86.9$ & $686.0 \pm 2.1$ & 43.49 & 43.46 & $<51.6$ & $<32.2^{* *}$ & 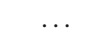 & \\
\hline NGC 4945 & $E^{*}$ & & $4.94 \pm 0.08$ & $4.66 \pm 0.02$ & & $936.4 \pm 24.0$ & & 41.80 & & 286.4 & & \\
\hline Centaurus A & $\mathrm{P}$ & $1.04 \pm 0.03$ & $0.91 \pm 0.01$ & $1.10 \pm 0.01$ & $1655.0 \pm 47.1$ & $1648.6 \pm 8.8$ & 41.98 & 41.98 & $<9.8$ & 37.2 & 20.2 & 8.1 \\
\hline NGC 5135 & E & $0.72 \pm 0.07$ & $1.01 \pm 0.04$ & $0.15 \pm 0.01$ & $126.7 \pm 4.0$ & $250.5 \pm 1.5$ & 43.27 & 43.57 & $<14.4$ & 384.9 & 31.0 & 26.4 \\
\hline Circinus & E & $1.33 \pm 0.09$ & $1.15 \pm 0.01$ & $0.73 \pm 0.01$ & $18617.0 \pm 1555.0$ & $15579.6 \pm 127.0$ & 43.15 & 43.07 & $<13.1$ & 39.3 & $<17.0$ & 9.6 \\
\hline NGC 5506 & $\mathrm{E}$ & $0.95 \pm 0.34$ & $0.80 \pm 0.01$ & $0.71 \pm 0.01$ & $1489.0 \pm 398.6$ & $1299.6 \pm 1.6$ & 43.73 & 43.67 & $<13.1$ & 36.6 & 13.6 & 36.3 \\
\hline NGC 5643 & E & $0.57 \pm 0.12$ & $0.67 \pm 0.02$ & $0.21 \pm 0.01$ & $277.1 \pm 37.0$ & $287.2 \pm 2.6$ & 42.53 & . & $<33.2^{* *}$ & 178.9 & 38.6 & 51.4 \\
\hline NGC 5728 & $\mathrm{P}$ & & $1.22 \pm 0.04$ & $1.10 \pm 0.01$ & & $109.6 \pm 1.7$ & & .. & $<87.4$ & 402.7 & $<43.7$ & 197.8 \\
\hline IC $4518 \mathrm{~W}$ & $\mathrm{P}$ & $1.54 \pm 0.19$ & $1.11 \pm 0.01$ & $1.10 \pm 0.02$ & $181.9 \pm 30.0$ & $283.7 \pm 1.0$ & 43.58 & & $<17.8$ & 104.6 & 41.2 & 64.2 \\
\hline ESO 103-G35 & $\mathrm{P}$ & $0.92 \pm 0.09$ & $0.83 \pm 0.01$ & $0.65 \pm 0.01$ & $578.4 \pm 34.0$ & $713.9 \pm 5.2$ & 43.91 & 44.00 & $<7.1$ & & 23.4 & 14.8 \\
\hline IC 5063 & E & $0.39 \pm 0.19$ & $0.32 \pm 0.02$ & $0.01 \pm 0.01$ & $627.8 \pm 127.4$ & $1081.8 \pm 2.9$ & 43.81 & 44.04 & $<26.8$ & & 22.0 & 20.8 \\
\hline NGC 7130 & E & $0.66 \pm 0.09$ & $0.87 \pm 0.03$ & $0.10 \pm 0.01$ & $148.9 \pm 10.7$ & $232.8 \pm 4.5$ & 43.49 & 43.80 & 103.8 & 225.7 & 10.9 & 15.1 \\
\hline NGC 7172 & E & $1.99 \pm 0.35$ & $1.87 \pm 0.03$ & $2.10 \pm 0.01$ & $156.2 \pm 13.9$ & $192.9 \pm 2.1$ & 42.89 & 42.98 & $<39.5^{* *}$ & 156.0 & 60.1 & 40.0 \\
\hline $3 \mathrm{C} 445$ & E & $-0.12 \pm 0.04$ & $-0.14 \pm 0.02$ & $0.00 \pm 0.01$ & $167.7 \pm 7.9$ & $171.4 \pm 3.1$ & 44.62 & 44.63 & $<22.9$ & & $<11.6$ & 4.6 \\
\hline NGC 7479 & $\mathrm{P}$ & $2.51 \pm 0.18$ & $2.00 \pm 0.03$ & $1.85 \pm 0.01$ & $532.7 \pm 77.1$ & $563.7 \pm 4.6$ & 43.42 & 43.44 & $<8.3$ & $13.1^{* *}$ & & 15.0 \\
\hline NGC 7582 & E & $1.17 \pm 0.21$ & $1.17 \pm 0.01$ & $0.85 \pm 0.01$ & $409.1 \pm 77.2$ & $1096.0 \pm 2.5$ & 42.87 & 43.30 & $<23.6^{* *}$ & 376.7 & $<5.4^{* *}$ & 43.8 \\
\hline
\end{tabular}

Notes. $\tau_{9.7}$ is measured in the $8-13 \mu \mathrm{m}$ band except when using PAHFIT in the Spitzer spectra for which we have used the full 5-35 $\mu \mathrm{m}$ band. (P) Point-like source and (E) Extended. ${ }^{(*)}$ NGC 4945 has no point-like component. Equivalent widths in units of $10^{-3} \mu \mathrm{m} .{ }^{(* *)}$ Tentative detection of $\mathrm{PAH}$ at $11.3 \mu \mathrm{m}$ or [SIV] at $10.5 \mu \mathrm{m}$ (1 sigma above the continuum level).

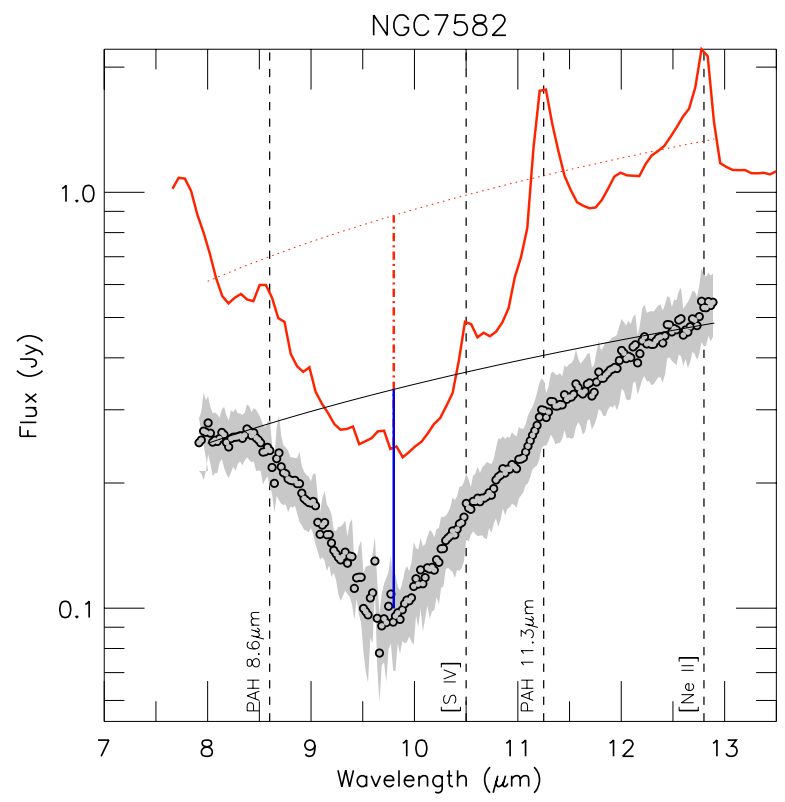

Fig. 3. T-ReCS (open dots) and Spitzer/IRS (red-solid line) spectra of NGC 7582. The errors are shown as a grey area. Interpolated continuum and estimated silicate feature depth are shown as a black-solid (red-dotted) line and vertical solid blue (red dotted-dashed) line for the T-ReCS (Spitzer/IRS) spectrum. Appendix A includes the spectra of the sample.

similar morphologies in all the filters. NGC 4945 is the only AGN without an unresolved point-like source. Overall, we find that the morphology of the nuclear source has no clear relation to the AGN type.

\subsection{MIR spectra}

Figure 3 shows the nuclear spectrum of NGC 7582. The spectra of the entire sample are included in appendix A. The shaded regions show the systematic errors of the extracted spectra. The spectra show systematic errors of less than $10 \%$ of the flux in all the objects except for NGC 5728, in which the error is much higher ( 40\%). We have excluded NGC 5728 from this spectral analysis due to the lack of high-quality data. Moreover, NGC 4945 is also excluded due to the lack of a nuclear component. Thus, our sample of nuclear spectra comprises 20 AGN. Our sample has three objects in common with the sample reported by Hönig et al. (2010a) using VISIR/VLT data (NGC 5643, IC 5063, and NGC 7582). The shape and detected features in the three VISIR spectra in common with our sample are consistent with those reported with T-ReCS. The total flux calibration is consistent with ours except for IC 5063, where we find a flux level twice fainter than reported by Honig.

The low spatial resolution spectra (Short-Low module) Spitzer spectra in the same spectral range as T-ReCS are also shown in the appendix when available. Spitzer data were retrieved from the Cornell Atlas of Spitzer/IRS Sources (CASSIS, Lebouteiller et al. 2011). These spectra are extracted using the optimal extraction region to ensure the best $\mathrm{S} / \mathrm{N}$. IC $4518 \mathrm{~W}$ was reduced with the Smart package ${ }^{13}$ (version 8.2.6, Higdon et al. 2004; Lebouteiller et al. 2010) since it was not available through CASSIS. There were Spitzer/IRS spectra for all the AGN in our sample except for NGC 3094.

The observed apparent optical depth at $\lambda \sim 9.7 \mu \mathrm{m}$ can be measured as the ratio between the expected continuum flux $\left(f_{9.7, \text { cont }}\right)$ and the observed flux in the feature $\left(f_{9.7, \mathrm{obs}}\right.$, see Shi et al. 2006; Spoon et al. 2007; Levenson et al. 2007; Georgantopoulos et al. 2011):

$\tau_{9.7}=\ln \left(f_{9.7, \mathrm{cont}} / f_{9.7, \mathrm{obs}}\right)$.

Sources with absorption (emission) features show positive (neg-

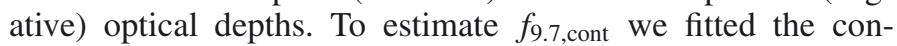
tinuum to a linear relation to the $8.5 \mu \mathrm{m}$ and $12.5 \mu \mathrm{m}$

${ }^{13}$ SMART was developed by the IRS Team at Cornell University. 


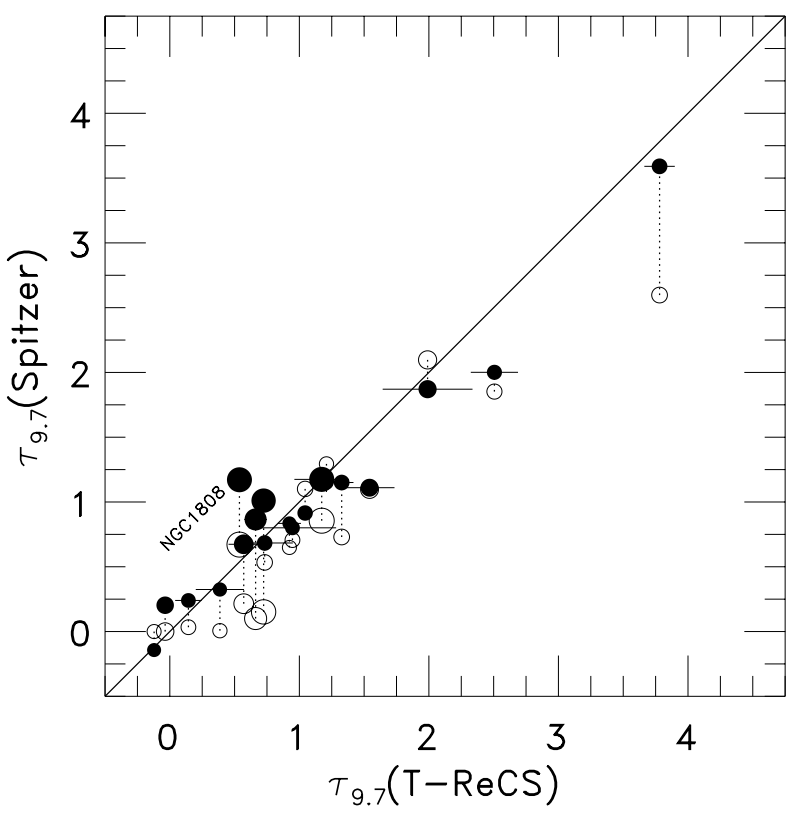

Fig. 4. Optical depth $\tau_{9.7}$ using Spitzer/IRS and T-ReCS data. Filled symbols show the optical depth $\tau_{9.7}$ using the linear interpolation in the $8-13 \mu \mathrm{m}$ band and empty symbols show the optical depth $\tau_{9.7}$ using the routine PAHFIT in the 5-35 $\mu \mathrm{m}$ band (linked by short dotted lines). The size of the symbols scales with the strength of the PAHs. The continuous line shows the one-to-one relation.

flux for each object. Figure 3 shows the continuum as a solid (dotted) thin line for T-ReCS (Spitzer) data. The $f_{9.7, \text { cont }}$ is estimated by the linear interpolation of this continuum flux at $9.7 \mu \mathrm{m}$. The error on the $\tau_{9.7}$ was determined by the $\mathrm{S} / \mathrm{N}$ of the continuum flux. More sophisticated methods, such as spline fit (Mason et al. 2012), PAHFIT (Smith et al. 2007), or DecompIR (Mullaney et al. 2011), are also useful for measuring $\tau_{9.7}$ especially when the AGN is contaminated by extranuclear emission. However, as discussed in the previous section, our sample is mostly dominated by AGN. Moreover, these other methods require wider wavelength ranges because they are based on a multiple component fitting and need a larger number of data points to avoid degenerating of the results. However, no strong PAH features are detected in our sample (see Sect. 5). The optical depth has also been measured for the Spitzer spectra with the same methodology. PAHFIT may turn out to be more appropriate for Spitzer data given its longer wavelength range. We did however, use the same method to measure $\tau_{9.7}$ as for the T-ReCS spectra for consistency in the comparison of the silicate measurements.

Strong PAHs could introduce uncertainties into the continuum determination using our linear interpolation (Spoon et al. 2007). To check this, we computed $\tau_{9.7}$ using PAHFIT in the full wavelength range $(5-35 \mu \mathrm{m})$ of the Spitzer/IRS spectra. Figure 4 shows $\tau_{9.7}$ for Spitzer spectra versus that of the T-ReCS spectra. Spitzer/IRS and T-ReCS data show similar $\tau_{9.7}$ using the linear interpolation. The mean difference between T-ReCS and Spitzer $\tau_{9.7}$ is $\left\langle\tau_{9.7}(\mathrm{~T}-\mathrm{ReCS})-\tau_{9.7}(\right.$ Spitzer $\left.)\right\rangle=0.03$. However, when we use a broader wavelength range and PAHFIT we recover shallower optical depths for the Spitzer/IRS spectra $\left(\left\langle\tau_{9.7}(\mathrm{~T}-\mathrm{ReCS})-\tau_{9.7}(\right.\right.$ Spitzer $\left.\left.)\right\rangle=0.28\right)$. Thus, it seems that there is a systematic error of $\sim 0.25$ between the two methodologies.

Only in two objects does the value of $\tau_{9.7}$ for the Spitzer/ IRS spectra show a higher value than found for T-ReCS, namely NGC 1808 and NGC 5135. These two sources show the largest
PAH features. In fact, we recover a lower value for both objects when using PAHFIT. In the following we use the $\tau_{9.7}$ obtained by the linear interpolation for consistency with the T-ReCS data, although in Sect. 5.1 we discuss how $\tau_{9.7}$ could affect detection of the PAH feature.

The apparent optical depths $\tau_{9.7}$ measured are included in Table 3. In NGC 3094 and NGC 7479, the bottom of the silicate absorption is very noisy so the determination of $\tau_{9.7}$ is poorly constrained. Thus, our measurement can be considered as a lower limit.

These T-ReCS data have already been presented in the literature for a small number of individual objects. The optical depth at $9.7 \mu \mathrm{m}$ of NGC 3281 has already been reported recently by Sales et al. (2011) using the same data. They found a value of $\tau_{9.7}=1.3 \pm 0.1$, in good agreement with our results. Díaz-Santos et al. (2010) also computed the silicate absorption of three objects using T-ReCS data and finding similar results.

Most of the Spitzer/IRS spectra give similar $\tau_{9.7}$ values to those measured from the T-ReCS data, although the former tend to be lower. When using PAHFIT and a broader wavelength range, these discrepancies become larger. This might reflect different dust configurations (see Sect. 5.1). Large discrepancies are found only for NGC 1808, NGC 5135, IC 4518W, and NGC 7479. For NGC 1808 and NGC 5135, the $\tau_{9.7}$ estimates are lower than the Spitzer/IRS results. These two sources show the most contaminated Spitzer spectra of the sample (strong PAH features). NGC 1808 shows a lower value when using PAHFIT, while NGC 5135 shows an even higher one.

We observed a wide range of silicate absorptions $\left(\tau_{9.7}=\right.$ $[-0.12,3.78])$ with a mean value and a standard deviation of $\left\langle\tau_{9.7}\right\rangle=1.20 \pm 1.05$. Only two sources show $\tau_{9.7}<0$, indicating silicate features in emission. Among them the silicate feature seen in emission for NGC 1365 is seen in absorption by Spitzer/IRS. The comparison with the literature is rather difficult due to the different criteria for determining the continuum. However, we have compared $\tau_{9.7}$ for Spitzer with the $12 \mu \mathrm{m}$ sample reported by $\mathrm{Wu}$ et al. (2009), finding a good agreement for the 8 objects in common.

The most significant features in the T-ReCS spectra are [SIV] at $10.5 \mu \mathrm{m}$, the PAH features at 8.7 and $11.3 \mu \mathrm{m}$, and [NeII] at $12.8 \mu \mathrm{m}^{14}$. We have measured the equivalent width $(E W)$ of [SIV] and PAH at $11.3 \mu \mathrm{m}$, dividing the integrated flux of the features by the adopted continuum near the feature ("feature" routine within IDL). We computed the upper limit of the $E W \mathrm{~s}$ to account for the error in the continuum. We have also obtained EWs for these lines using Spitzer spectra. These EWs are also included in Table 3 . The continuum luminosity at $12 \mu \mathrm{m}$, $v L_{v}(12 \mu \mathrm{m})$, obtained for T-ReCS and Spitzer, is also included in Table 3.

\section{Contributors to the MIR spectra of AGN}

\subsection{The $11.3 \mu \mathrm{m} P A H$ feature}

The presence of PAHs has been associated to regions of star formation, given the global correlations between star formation activity and PAH strength (e.g. Roche et al. 1991; Smith et al. 2007; Wu et al. 2009; Diamond-Stanic \& Rieke 2010). This emission is thought to originate in photo-dissociation

\footnotetext{
14 The short wavelength range prevents us from confirming the presence of the PAH feature at $8.7 \mu \mathrm{m}$. Moreover, we have not attempted to measure the $[\mathrm{NeII}]$ line because in most cases it is too close to the limit of the wavelength range.
} 
regions (PDRs) where aromatic molecules are shielded from the radiation field produced by hot stars.

It has been proposed that the hard radiation field produced by the AGN may destroy the PAH molecules (Genzel et al. 1998; Siebenmorgen et al. 2004), as indicated by the decrease in the $E W(\mathrm{PAH} 11.3 \mu \mathrm{m})$ with infrared colours (Wu et al. 2009). Nonetheless, Diamond-Stanic \& Rieke (2010) find $11.3 \mu \mathrm{m}$ emission in most of their AGN in a sample of 35 Seyferts observed with Spitzer on scales of $1 \mathrm{kpc}$ and find suppression of the PAH features only for the 6.2, 7.7, and $8.6 \mu \mathrm{m}$ PAHs (see also Diamond-Stanic \& Rieke 2012).

A strong PAH feature at $11.3 \mu \mathrm{m}$ is present only in NGC 1808 and NGC 7130 in the T-ReCS spectra. A hint of the PAH feature is also present in other four AGN (NGC 1386, NGC 5643, NGC 7172, and NGC 7582). Thus, for 14 out of 20 sources, their high spatial resolution T-ReCS spectra are free of signs of star formation in the MIR, as traced by the PAHs.

From visual inspection of the 2D T-ReCS spectra of these sources, the PAH feature is spatially extended for NGC 1808 and coincident with the nuclear emission for NGC 7130. Moreover, the central UV continuum of NGC 7130 consists of several knots of star formation distributed in an asymmetric ring within 1 arcsec, not spatially resolved by our data (González Delgado et al. 1998). Thus, the PAH features detected in NGC 7130 could be associated to this ring. NGC 1808 shows a circumnuclear starburst region (e.g. Collison et al. 1994), together with an AGN (Veron-Cetty \& Veron 1985). The $3.3 \mu \mathrm{m}$ PAH emission of NGC 1808 was studied by Tacconi-Garman et al. (2005) using high spatial resolution images obtained with the ISAAC (VLT) instrument. They found PAH emission coincident with the nucleus to support these results.

Wu et al. (2009) describe a method of estimating the AGN contribution when the PAH feature at $11.3 \mu \mathrm{m}$ is present. It is based on the constant ratio between the flux of the PAH feature and the continuum at $12 \mu \mathrm{m}\left(F_{12 \mu \mathrm{m}, \mathrm{SF}} \simeq 22.73 \times F_{\mathrm{PAH}}\right)$ where the fraction of the AGN at MIR is $F_{\mathrm{AGN}}=\left(F_{12 \mu \mathrm{m}}-\right.$ $\left.F_{12 \mu \mathrm{m}, \mathrm{SF}}\right) / F_{12 \mu \mathrm{m}}$. We computed this value for the two objects with the strongest PAH emissions and find 87\% for NGC 1808 and $93 \%$ for NGC 7130. Therefore, the AGN contribution seems to be high even for the objects with the strongest PAH features. However, this method shows a $25 \%$ error (see Sect. 5.3 for further analysis of the AGN contribution for these two sources).

Goulding et al. (2012) used Spitzer/IRS spectra to find that star formation dominates in most Compton-thick AGN. They argue that this might be an intrinsic characteristic of heavily absorbed AGN. Our sample includes eight Compton-thick AGN, but only NGC 7130 shows PAH emission in the T-ReCS spectra. However, as explained above, the T-ReCS spectrum of NGC 7130 includes an unresolved ring of star formation. Thus, it seems that the nuclear star formation among Comptonthick AGN might be as uncommon as for the rest of the AGN. However, this needs to be confirmed for a larger sample of Compton-thick AGN.

The lack of PAHs in the nuclear spectral spectra of AGN has already been claimed by Siebenmorgen et al. (2004) using TIMMI2 spectra. Hönig et al. (2010a) also report a lack of PAH features in their high spatially resolved VISIR spectra, detecting some minor emission of PAH at $11.3 \mu \mathrm{m}$ only in five case. (In the two objects in common we also detect this tentative PAH emission.)

When we compare the T-ReCS and the Spitzer/IRS spectra for those objects with strong PAH detected with T-ReCS (i.e. NGC 1808 and NGC 7130), the Spitzer PAH $11.3 \mu \mathrm{m}$ feature is indeed present. Besides this, the PAH feature is detected in 16 other objects. The Spitzer spectra are free of PAH emission lines in only four objects named NGC 3281, ESO 103G35, IC 5063, and 3C 445. It seems that in most cases the $\mathrm{PAH}$ emission comes from emitting regions outside of 7-130 pc (depending on the distance) to the AGN. Considering that the Spitzer spectra have a spatial scale of 3 arcsec, that extension corresponds to anything between $0.6-0.8$ arcsec in T-ReCS (depending on observation) and the 3 arcsec used for Spitzer spectra. This is consistent with previous studies that suggest that AGN lack strong PAH features (Genzel et al. 1998; Hernán-Caballero et al. 2009; Hönig et al. 2010a).

The PAH at $11.3 \mu \mathrm{m}$ is, however, embedded in the silicate absorption feature and thus in cases of high extinction the PAH feature could be attenuated (e.g. Brandl et al. 2006; Pereira-Santaella et al. 2010a). According to Smith et al. (2007), the optical depth at $11.3 \mu \mathrm{m}$ is related to the one at $9.7 \mu \mathrm{m}$ as $\tau_{11.3 \mu \mathrm{m}}=0.44 \times \tau_{9.7 \mu \mathrm{m}}$. For optical depths of $\tau_{9.7 \mu \mathrm{m}}=$ $[1,2,3]$ (common optical depths in our sample, see Sect. 6), the intensity of the line could be reduced by a factor of $I=$ $I_{\mathrm{O}} \times[0.64,0.41,0.26]$. Thus, the attenuation of the PAH emission feature is expected to be large in some cases. Since the optical depths measured in our sample are similar for both T-ReCS and Spitzer spectra, we would expect that the PAHs must be attenuated in both. However, using PAHFIT for Spitzer spectra (see Sect. 4.2), the $\tau_{9.7}$ optical depths are shallower in the Spitzer spectra than in T-ReCS. Thus, the lack of PAH features in the latter could be the result of the extra attenuation suffered by the T-ReCS spectra. This attenuation can be expressed as $I=I_{0} \times \exp \left(-0.44\left(\tau_{9.7}+\Delta \tau_{9.7}\right)\right)$, where $\Delta \tau_{9.7}=$ $\tau_{9.7}(\mathrm{~T}-\mathrm{ReCS})-\tau_{9.7}($ Spitzer $)$ is the difference between the attenuation measured using T-ReCS and Spitzer spectra. Using the values of $\tau_{9.7}$ given in Table 3 , in most of the cases this extra attenuation cannot explain the lack or weakness of the $11.3 \mu \mathrm{m}$ PAH seen in T-ReCS data. In particular, the upper limit on the $E W(\mathrm{PAH}$ at $11.3 \mu \mathrm{m})$ for T-ReCS data could be explained as the result of the extra attenuation only in two galaxies, namely NGC 1386 and NGC 7479. Thus, the attenuation of the spectrum might not be responsible for the lack of PAH features in AGN.

This lack of PAH emission in the proximity of the AGN $(<100 \mathrm{pc})$ indicates that the AGN emission is able to destroy the PAH molecules (Wu et al. 2009; Mason et al. 2007). Alternatively, the $\mathrm{BH}$ growth can continue after there is no longer enough gas fuel to maintain the star formation activity (see the numerical simulations given by Hopkins 2012). These delays have also been detected observationally (e.g. Davies et al. 2007). Therefore, the lack of PAH emission might be the result of a delay between the onset of the star formation rate and the black hole $(\mathrm{BH})$ accretion rate.

It is not easy to disentangle which of these two possibilities is the main reason for the lack of PAHs in AGN. If the PAHs are destroyed by the AGN, we would expect that PAHs may survive in the vicinity of low-luminosity AGN. In support of this, we detected PAH features in the nuclear region of NGC 1808, which is the object in our sample with the lowest X-ray luminosity. Hönig et al. (2010a) find that most AGN show a lower continuum in the VISIR than in the Spitzer data when the PAH are suppressed. They argue that favours of the lack of star formation as the main reason for the suppression of PAHs because the continuum of the MIR spectrum is roughly constant between VISIR and Spitzer/IRS if the PAH destruction is an effect of grain destruction. In our sample, although the continuum is fairly constant for some of the objects (e.g. Centaurus A), the continuum suppression in the T-ReCS data is clear in some of the other objects (e.g. NGC 7582). 


\subsection{The [S IV] emission line at $10.5 \mu \mathrm{m}$}

Another widely used AGN diagnostic line is the [SIV] at $10.5 \mu \mathrm{m}$ since this line is thought to have originated in the narrow line region (NLR) (Dasyra et al. 2011, and references therein). However, the [SIV] at $10.5 \mu \mathrm{m}$ line arises from ions with an ionization potential of $35 \mathrm{eV}$, similar to the optical line [OIII] at $5007 \AA$ (see Trouille et al. 2011). Thus, this line can be produced in star-forming regions, as well as in AGN (Pereira-Santaella et al. 2010b).

The [SIV] line is detected in $11 \mathrm{AGN}$, and a hint of this line is also present in two other sources in the T-ReCS data (NGC 3281, and NGC 7582). In all these cases it is also detected with Spitzer. Interestingly, Spitzer detected this line in five other additional sources (NGC 1365, NGC 1808, Circinus, $3 \mathrm{C} 445$, and NGC 7479) ${ }^{15}$. The lack of this line in these objects could be due to the low $\mathrm{S} / \mathrm{N}$ of the T-ReCS spectra. In fact, they are the faintest emission lines, according to their $E W([\mathrm{SIV}])$ in the Spitzer spectra. Besides, only NGC 1365 shows a limit that is inconsistent with the $E W$ reported with Spitzer data. Thus we claim that the origin of the [SIV] line is consistent with being nuclear in our sample (see also Pereira-Santaella et al. 2011).

\subsection{X-ray to MIR relation}

The MIR emission in AGN is produced in the reprocessing of the soft X-ray and UV radiation by dust located in the torus. At the same time, escaping high-energy photons could destroy PAH molecules at sub-kpc scales (Voit 1992). This is supported by the lack of PAH emission in the T-ReCS spectra (see Sect. 5.1). The reprocessed dust emission should then produce a significant correlation between X-ray luminosity (after correction from X-ray absorption) and MIR luminosity. This correlation has already being found by other authors (Horst et al. 2008; Levenson et al. 2009; Gandhi et al. 2009; Hönig et al. 2010a; Ichikawa et al. 2012).

Figure 5 shows the absorption-corrected 2-10 keV luminosity $L_{2-10 \mathrm{keV}}$ versus the $12 \mu \mathrm{m}$ continuum luminosity $L_{12} \mu \mathrm{m}$. $\mathrm{X}$-ray errors represent the expected X-ray variability among AGN of 0.5 dex while the MIR errors are inferred from the photometric errors including $10 \%$ error for the total flux calibration. Spitzer luminosities agree very well with the T-ReCS luminosities for most of the sources $\left(\log \left(L_{12, \mathrm{~T}-\mathrm{ReCS}} / L_{12, \text { Spitzer }}\right)<0.4\right)$.

Most of the objects follow the same correlation as found for AGN by Levenson et al. (2009) and Asmus et al. (2011). Moreover, those objects with significantly higher MIR emission compared to X-rays are also far from the correlation found by Asmus et al. (2011) for starburst galaxies. The X-ray luminosity of NGC 7479 is not corrected for absorption although the object is Compton-thick. Thus, its X-ray luminosity could be up to 60 times higher than reported here (see Panessa et al. 2006), in better agreement with the correlation for AGN. Interestingly, NGC 1808 (the closest to the starburst correlation) shows the strongest PAH emission in our T-ReCS sample, indicating that the AGN component of this source does not dominate the MIR emission. The locus of this object in the correlation indicates that the fraction of star formation must be higher than the estimated $87 \%$, see Sect. 5.1. In fact, the existence of an AGN in the nucleus of NGC 1808 has been under debate (e.g. Phillips 1993; Awaki et al. 1996). Jiménez-Bailón et al. (2005)

\footnotetext{
15 This line is also detected in NGC 5728, but the T-ReCS spectra show a very low $\mathrm{S} / \mathrm{N}$ (see Sect. 4.2).
}

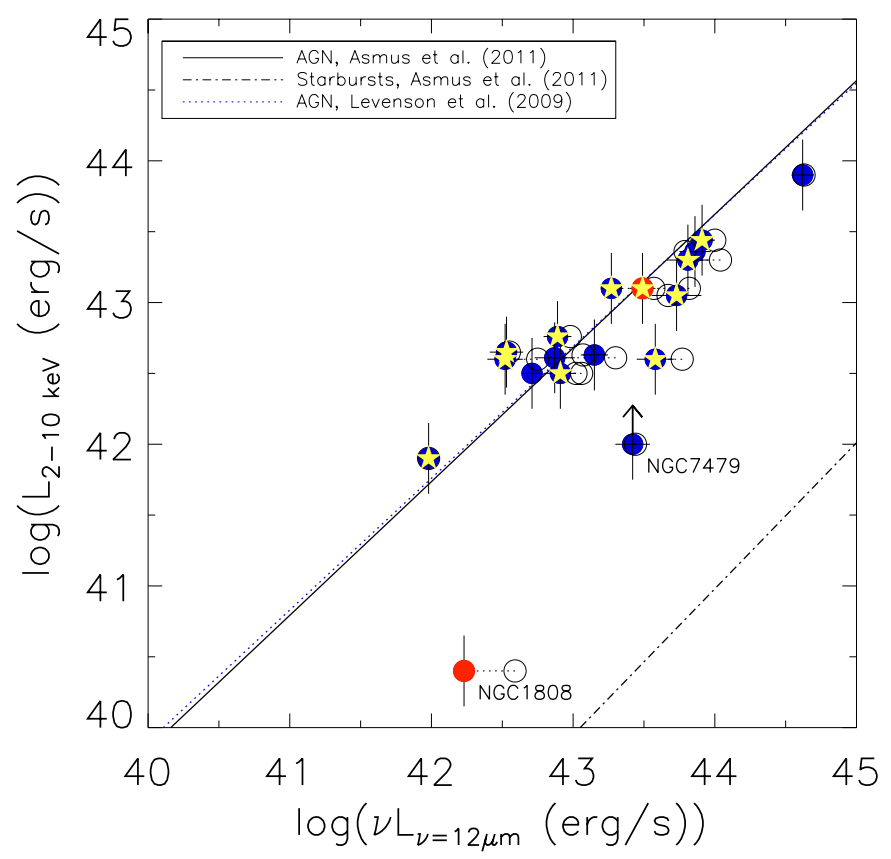

Fig. 5. Absorption corrected $2-10 \mathrm{keV}$ luminosity versus $12 \mu \mathrm{m}$ luminosity, both in logarithmic units. Filled circles are T-ReCS data and open circles Spitzer data. The dotted lines link Spitzer to T-ReCS data for the same object. Yellow stars and red circles mark the objects with the strongest [SIV] $10.5 \mu \mathrm{m}$ and PAH at $11.3 \mu \mathrm{m}$ emissions in the T-ReCS spectra, respectively. The blue dotted line shows the best fit found by Levenson et al. (2009) for AGN. Black dotted-dashed and solid lines represent the best fit found by Asmus et al. (2011) for Starburst galaxies and AGN, respectively.

studied the X-ray emission of NGC 1808 with XMM-Newton and Chandra showing the co-existence of thermal diffuse plasma and non-nuclear unresolved point-like sources associated with the starburst activity, together with a low-luminosity AGN. However, it is clear from our correlation that the AGN in NGC 1808 does not dominate the MIR emission.

Thus, despite the heterogeneous sample and the large number of highly obscured objects, the $12 \mu \mathrm{m}$ luminosity and the $\mathrm{X}$-ray luminosities are still well correlated. Thus, except for the nucleus in NGC 1808, the AGN dominates the continuum emission at MIR frequencies.

\section{The silicate absorption/emission feature}

Wu et al. (2009) used Spitzer/data to find that the silicate feature in Type-1 Seyferts is rather weak, while in Type-2 Seyferts, it is more likely to display strong silicate absorption.

In the T-ReCS spectra, only the spectra of NGC 1365 and 3C 445 show silicate features in emission while NGC 3081 shows very little absorption (see Appendix A and $\tau_{9.7}$ in Table 3 ). The other sources (i.e. 17 out of 20) show very strong silicate absorptions. The two objects showing the silicate feature in emission, NGC 1365 and 3C 445, are Type 1.5 AGN. All the Compton-thick AGN in our sample ubiquitously show strong silicate absorption features. This contrasts with Goulding et al. (2012) who find that most of the Compton-thick AGN in their sample do not show such strong silicate absorption. However, since our sample is not complete, the statistics may well be biased by the selection of the individual observing proposals of the targets in our sample. In particular none of 


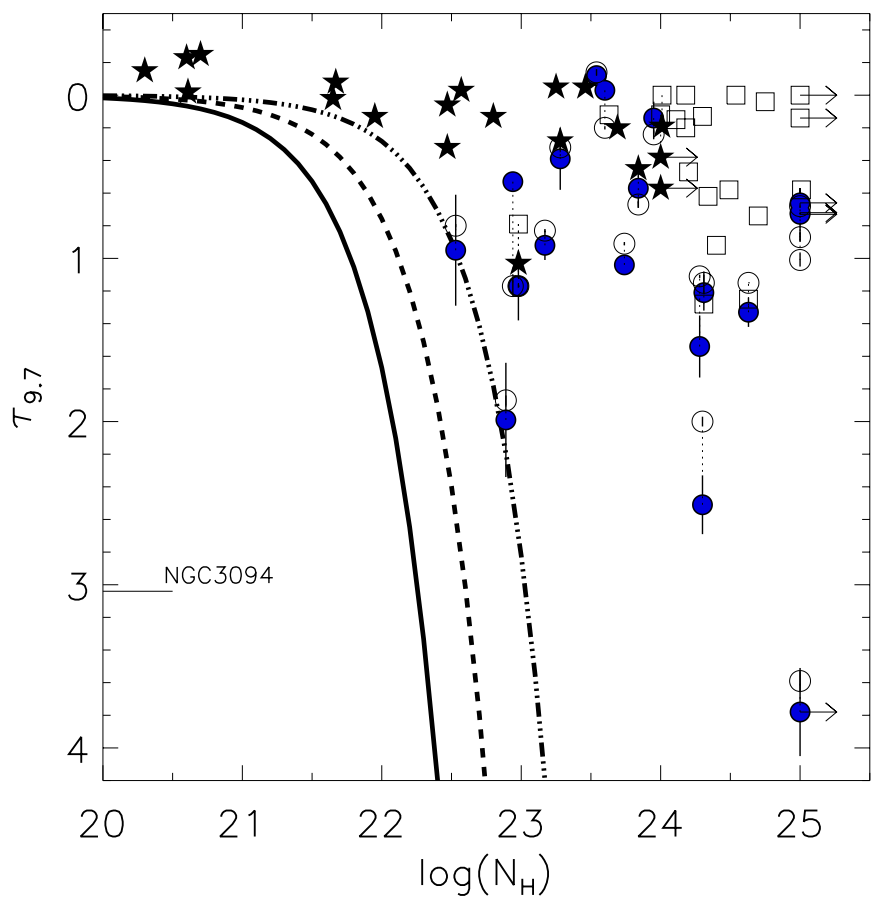

Fig. 6. Optical depth at $9.7 \mu \mathrm{m}, \tau_{9.7}$ versus logarithmic of the hydrogen column density at X-rays, $N_{\mathrm{H}} . N_{\mathrm{H}}$ in units of $\mathrm{cm}^{-2}$. T-ReCS data are shown as blue-filled circles and the Spitzer/IRS data included in our work are shown as empty circles. The object without reported X-ray measurement is labelled as a short line in the $Y$-axis. Low limits on the $N_{\mathrm{H}}$ are shown as right arrows. Compton-thick sources reported by Goulding et al. (2012) are shown as open squares, and AGN observed with VISIR/VLT by Hönig et al. (2010a) are shown as black-filled stars. Constant gas-to-dust ratio (i.e. the uniform dusty torus) assuming the viewing angles of $i=0,40,80^{\circ}$ are shown with the continuous, dashed, and dot-dashed lines, respectively.

the Compton-thick AGN with low optical depth reported by Goulding et al. (2012) have been observed by T-ReCS, while we do share three Compton-thick sources with strong silicate absorption features ${ }^{16}$.

The X-ray hydrogen column density $\left(N_{\mathrm{H}}\right)$ of 20 of the sources reported in the literature are shown in Table $1^{17}$. Figure 6 shows $\tau_{9.7}$ versus $N_{\mathrm{H}}$ on logarithmic scale. For comparison purposes we have included the high angular resolution data for AGN observed with VISIR/VLT (Hönig et al. 2010a), as well as the Compton-thick AGN observed with Spitzer/IRS from the sample of Goulding et al. (2012). The filled symbols are chosen to emphasize high spatial resolution data (i.e. T-ReCS and VISIR).

If we focus on the high spatial resolution data we find a trend in the sense of increasing $\tau_{9.7}$ for higher $N_{\mathrm{H}}$. This relation has already been seen by several authors (e.g. Shi et al. 2006; Wu et al. 2009; Hönig et al. 2010a; Alonso-Herrero et al. 2011). Moreover, for $\log \left(N_{\mathrm{H}}\right)>23$ the silicate absorption shows a wide range of values from low or no absorption to deep silicate absorptions, in agreement with the results presented by $\mathrm{Wu}$ et al. (2009). In the next sections we try to model this relationship under two assumptions: (1) the uniform dusty torus model and (2) the clumpy torus models.

\footnotetext{
${ }^{16}$ We have five sources in common with Goulding et al. (2012), although two of them did not have high S/N T-ReCS data for the spectral analysis.

17 There are no X-ray data published for NGC 3094.
}

\subsection{Uniform dusty torus}

On the basis of a uniform (smooth), heavily-obscuring, subparsec scale torus (Pier \& Krolik 1992, 1993; Granato \& Danese 1994; Granato et al. 1997), the gas/dust-rich torus is responsible for both the MIR and the X-ray gas absorption through the gasto-dust relation. The constant gas-to-dust ratio can be estimated by assuming $N_{\mathrm{H}} / A_{V}=1.93 \times 10^{21} \mathrm{~cm}^{-2}$ and $\tau_{9.7}=0.07 \times \tau_{\mathrm{X}}$ (Draine \& Li 2007), where $\tau_{\mathrm{X}}$ is the total optical depth in X-rays (Fig. 6). For very low values of $N_{\mathrm{H}}$ it predicts no absorption, close to the actual values. Some objects, however, do show the silicate feature in emission, which cannot be explained with these models. Nonetheless, as already shown by Goulding et al. (2012), this simple model is far from the measurements for most of the objects.

While the MIR emission is integrated over the whole dusty torus, the X-ray absorption is only due to the amount of absorbing column density along the LOS. The influence of the $N_{\mathrm{H}}$ within the LOS can be estimated by assuming a density profile at the viewing angle $i$ (angle between the LOS and the plane of the torus). Nenkova et al. (2008a) assume a Gaussian distribution $e^{-\frac{i^{2}}{\sigma^{2}}}$, where $\sigma$ is the width of the toroidal distribution. The constant gas-to-dust ratio for $i=[0,40,80]^{\circ}$ degrees is shown for $\sigma=45^{\circ}$ (Fig. 6). Only two of the sources could be explained by this relation (NGC 7172 and NGC 5506), when assuming a highly inclined torus. Thus the observed $\tau_{9.7}$ feature is much shallower than predicted by this constant gas-to-dust relation. This has already been mentioned by Georgantopoulos et al. (2011), who show that although $\sim 70 \%$ of the sources with high optical absorption at $9.7 \mu \mathrm{m}$ are classified as Comptonthick AGN, the opposite is not true, since many Compton-thick sources do not show significant absorption at $9.7 \mu \mathrm{m}$ (see Fig. 6).

\subsection{Clumpy torus}

The clumpy torus models of Nenkova et al. (2008a,b) predict the silicate feature in emission even for high values of the optical depths of the individual clouds $\left(\tau_{\mathrm{V}}>100\right)$. This effect can also explain the low silicate absorption observed for some Comptonthick sources in our sample. In order to test this possibility, we used the libraries of CLUMPY ${ }^{18}$ models, a code modelling the AGN clumpy dust torus emission (Nenkova et al. 2008a,b). We obtained a set of SEDs for $\sigma=45^{\circ}, Y=R_{\text {out }} / R_{\text {inner }}=200$ (where $R_{\text {inner }}$ and $R_{\text {out }}$ are the inner and outer radii of the torus, respectively), an exponential slope of the radial distribution of clouds $q=2$ (see Nenkova et al. 2008b, for more details), $\tau_{V}=5-150$, and the number of clouds along the equator of the torus $N_{\mathrm{o}}=2-25$ clouds. We chose $Y$ to use the lowest maximum temperature and therefore the maximum silicate depth. Hereinafter we assume $\sigma=45^{\circ}$. Alonso-Herrero et al. (2011) has measured $\sigma=[30-60]^{\circ}$ for a sample of Type-2 Seyferts using a Bayesian method to fit the CLUMPY models (see also Asensio Ramos \& Ramos Almeida 2009). This parameter is not crucial since the silicate absorption is insensitive to the angular size of the torus when the LOS incidence angle of the ray is smaller than or close to the size of the torus (i.e. Type-2 Seyferts, see Nenkova et al. 2008b). Under this approximation, the total optical depth produced by the torus is the sum of the optical depths of the individual clouds along the $\operatorname{LOS}\left(\tau_{\mathrm{X}}=N \times \tau_{V}\right.$, where $N$ is the number of clouds along the LOS) and the number of clouds $N$, similar to the classical torus, is

$N=N_{\mathrm{o}} e^{-\frac{i^{2}}{\sigma^{2}}}$.

${ }^{18}$ http://www.pa.uky.edu/clumpy 


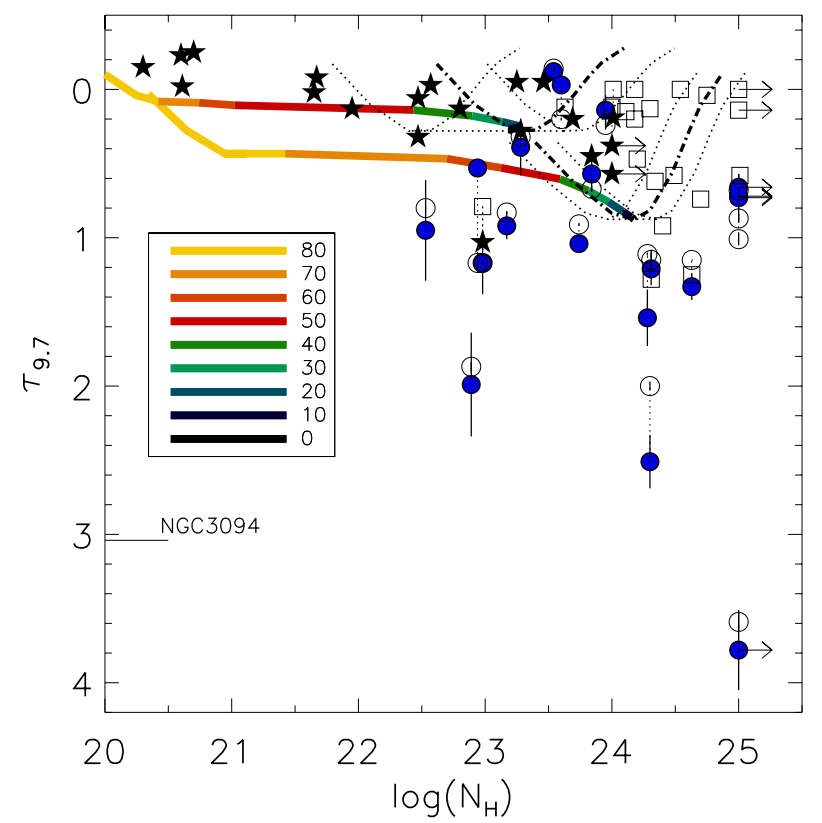

Fig. 7. Optical depth at $9.7 \mu \mathrm{m}, \tau_{9.7}$ versus logarithmic of the hydrogen column density at X-rays, $N_{\mathrm{H}} . N_{\mathrm{H}}$ in units of $\mathrm{cm}^{-2}$. Symbols as in Fig. 6. Dot-dashed lines are the CLUMPY model predictions for $i=0^{\circ}$ and $N_{\mathrm{o}}=[5,25]$ clouds. Dotted lines show how the curves evolve for the minimum and maximum number of clouds with a probability higher than $10 \%$ of intercepting the LOS. The coloured thick line show how the curve evolves when the viewing angle changes from $i=0^{\circ}$ (black) to $i=80^{\circ}$ (yellow). The dotted lines are the model that includes the Poisson probability of detecting a number of clouds along the ray (see text).

We have applied the same technique as described in Sect. 4.2 to obtain the silicate feature strength for these SEDs. Figure 7 shows $\tau_{9.7}$ for these models for an inclination $i=0$ (i.e. along the plane of the torus) and $N_{\mathrm{o}}$ of 5 and 25 clouds.

Nonetheless, this number of clouds $N$ is still an estimate of the mean number of clouds along the LOS. Statistically for a viewing angle $i$ and a mean equatorial number of clouds $N_{\mathrm{o}}$ the ray might intercept a range of clouds that might differ substantially from the average. We have estimated the range of $N$ following the prescription given by Nenkova et al. (2008a, see their appendix). Using Poissonian distributions, the probability that a ray intercepts a number of clouds $k$ normalized to the probability to intercept the mean value of clouds $N$ is

$\frac{P_{k}}{P_{N}}=\frac{N !}{k !} N^{(k-N)}$,

where the range of the number of clouds $(k)$ is obtained as the minimum and maximum for which the probability is smaller than $10 \%$. Higher (lower) $N_{\mathrm{H}}$ in Fig. 7 are the largest (lowest) number of clouds with a probability above $10 \%$.

Many of the Compton-thick AGN are well within the boundaries of this model if we are observing them close to the plane of the torus and the number of clouds is high $\left(N_{\mathrm{o}} \sim 5-25\right)$. The coloured thick lines show how this model changes with the inclination angles for the two $N_{\mathrm{o}}$. The model reproduces all the mildly obscured X-ray objects well (with $N_{\mathrm{H}}<10^{22} \mathrm{~cm}^{-2}$ ) when assuming a mean number of clouds of $N_{\mathrm{o}}>5$. This is in good agreement with the parameters of the Bayesian fit to CLUMPY models reported by Ramos Almeida et al. (2009, 2011), and Alonso-Herrero et al. (2011).

However, the model fails to reproduce a dozen sources with a high value for $\tau_{9.7}$. Nenkova et al. (2008a) show that, irrespective of the optical depth, the absorption feature produced by a clumpy torus is never deep $\left(\tau_{9.7}<1\right)$. This is confirmed by other clumpy models (e.g., Hönig \& Kishimoto 2010b). Alonso-Herrero et al. (2011) also note that it is possible that for the Seyfert galaxies with the deepest silicate features a clumpy medium in a toruslike configuration may not be appropriate to explain the observations. Levenson et al. (2007) show that deep silicate features require that the source is embedded in dust that is both optically and geometrically thick. They show that a geometrically thick shell structure is able to produce $\tau_{9.7}$ as high as 4 . One possibility, then, is that these objects show dust screen geometries in addition to the clouds of the clumpy torus.

\subsection{Contribution from extended dust to the observed silicate feature}

On the basis of the uniform dust torus models produced from radiative transfer theory (Dullemond \& van Bemmel 2005; Schartmann et al. 2005; Fritz et al. 2006), the MIR spectrum of obscured AGN included in our sample are expected to be dominated by a power law and significant silicate absorption features at $\lambda \sim 9.7 \mu \mathrm{m}$. However, as discussed in the previous sections, many AGN in our sample show silicate absorption features that are shallower than predicted for the homogeneous dusty model and often deeper than predicted for the clumpy model. Several authors have claimed that the dust seen at MIR frequencies might not be co-spatial with the torus gas measured at X-rays (Sturm et al. 2005; Goulding et al. 2012). In particular, the inclination angle of the host galaxy, dust lanes, or galaxy minor or major mergers may have significant effects on the AGN emission at MIR (Malkan et al. 1998; Matt et al. 2000; Deo et al. 2007, 2009; Alonso-Herrero et al. 2011; Goulding \& Alexander 2009; Goulding et al. 2012). Although not many indications of extended contributors to the emission can be seen in our spectra, most of the sources in our sample are extended (see Sect. 4.1). In this section we discuss the possible contribution of the host galaxy to the optical depth at $9.7 \mu \mathrm{m}$.

To study a possible extranuclear origin for the silicate absorption feature we looked for the superb spatial resolution that HST can achieve. Broad band HST images are available in the MAST archive $^{19}$ for $12 \mathrm{AGN}$ in our sample (10 with the $F 606 \mathrm{~W}$ filter and 2 with the F814W filter). Narrow-band filters have been used in five cases whenever broad-band filter images were not found. For the remaining five objects without HST images, we used the available catalogue of images in NED ${ }^{20}$ (NGC 3094, NGC 4418, Circinus, IC 4518W, and 3C 445). We compiled the axial ratio of the galaxies as the $b / a$ ratio, where $b$ and $a$ are the minor and major axes, respectively, to measure the inclination of the host galaxy (see Table 1). Axial ratios of $b / a<0.5$ are considered as edge-on galaxies. Dust lanes or extended dust across the nucleus are present in eight objects and the AGN is hosted in edge-on galaxies in nine objects. In ten objects no particular inclination or dust features are found. We have also checked for the morphological properties of the host galaxies of the sample. Five galaxies are in mergers, namely NGC 4945 (Aalto et al. 1991), Circinus (Aalto et al. 1991), IC 4518W (Vorontsov-Velyaminov 1977), NGC 7130 (Aalto et al. 1991), and NGC 7479 (Laine \& Heller 1999; Martin et al. 2000). These properties are included in Table 1. For comparison purposes, the same properties were looked for in the Goulding et al. (2012) and Hönig et al. (2010a) samples.

\footnotetext{
19 http://archive.stsci.edu/

${ }^{20}$ http://ned.ipac. caltech.edu
} 


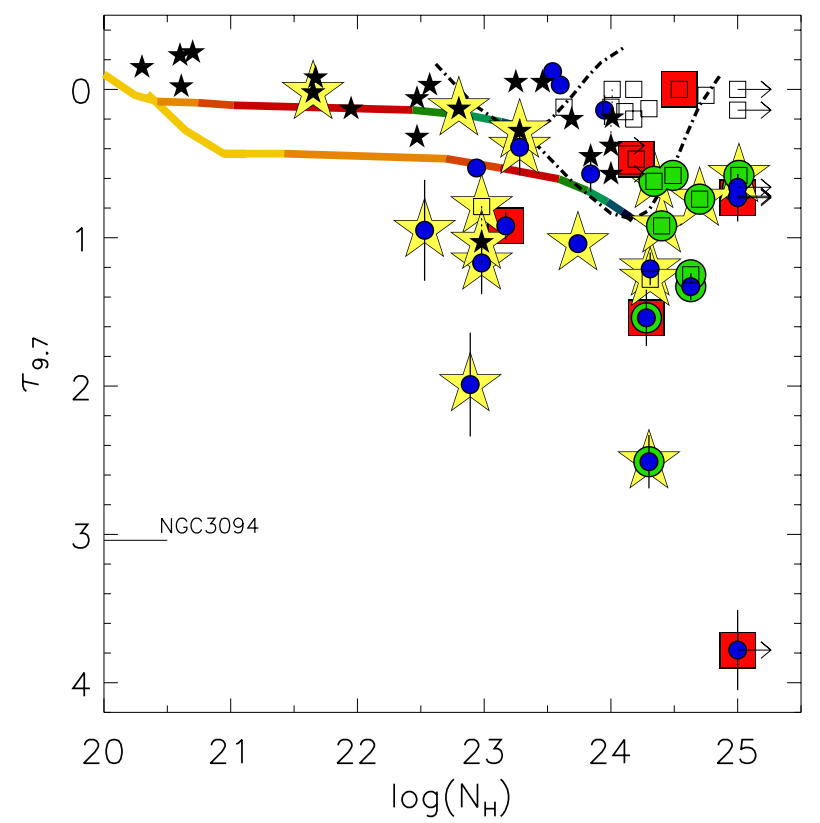

Fig. 8. Optical depth at $9.7 \mu \mathrm{m}, \tau_{9.7}$ versus logarithmic of the hydrogen column density at X-rays, $N_{\mathrm{H}}$. $N_{\mathrm{H}}$ in units of $\mathrm{cm}^{-2}$. Spitzer/IRS data of our sample are removed here for clarity. Edge-on galaxies are shown as big red squares, galaxies with dust lanes are shown with big yellow stars, and mergers are shown as big green circles. Edge-on galaxies are shown only when dust-lanes are not present for clarity of the plot.

Figure 8 shows $\tau_{9.7}$ versus $\mathrm{N}_{\mathrm{H}}$ where we mark those AGN hosted in edge-on galaxies, AGN with nuclear dust lanes, and mergers. For clarity we show only edge-on galaxies without nuclear dust-lanes. We note that all the objects with high $\tau_{9.7}$ are hosted in galaxies meeting one or more of these three properties. In general, clumpy torus models can only explain face-on galaxies free of either dust lanes and/or participating in mergers, although this analysis cannot rule out the host galaxy as the one responsible for the observed $\tau_{9.7}$, even for low $\tau_{9.7}$. Only three objects show one of these properties without a strong $\left(\tau_{9.7}<0.4\right)$ silicate absorption feature (IC 4329A, NGC 3227, and NGC 424). Therefore, we conclude that even at the high angular resolutions $(100 \mathrm{pc})$ of our observations, dust in the host galaxy can play a role in the overall dust extinction seen at MIR frequencies, and it does particularly when deep silicate absorption features are observed.

\section{Conclusions}

Here we present an analysis of 20 high spatial resolution MIR AGN spectra observed with T-ReCS (Gemini Observatory) on scales of $\sim 100 \mathrm{pc}$. The T-ReCS data have been processed with the pipeline RedCan. RedCan was developed by our group for the data reduction of imaging and spectroscopic data from the CanariCam instrument, recently commissioned at the GTC (La Palma, Spain). This data set has been complemented with Spitzer spectra probing kpc scales, as well as X-ray information for the sources. The main results of the study are:

1. The AGN contribution to the MIR spectrum on scales of $\sim 100 \mathrm{pc}$. We find that the AGN dominates the MIR emission as seen in the high-spatial resolution T-ReCS spectra presented here. This agrees with the relatively faint $11.3 \mu \mathrm{m}$ PAH emission observed in most of the AGN studied here.

2. The origin of the silicate feature. The observed strength of the nuclear (a few hundred pc) $9.7 \mu$ m optical depth could be well fitted with clumpy models with an $\mathrm{N}_{\mathrm{o}} \sim 5-25$ number of clouds, but only for sources with little mild silicate absorption. However, sources with the deepest $9.7 \mu \mathrm{m}$ optical depths cannot be reproduced with clumpy models. In these cases we have found that the host galaxies contribute to the nuclear component since they are detected in objects showing optical dust lanes and/or they are involved in a merger process.

3. The importance of the spatial resolution. Intense $11.3 \mu \mathrm{m}$ PAH feature emission is detected in most of the Spitzer/IRS spectra. This indicates that, at least in our sample of AGN, most of the star formation activity, as probed by this tracer, takes place in regions at least 100 pc away from the AGN. Interestingly, in most of the AGN, the $12 \mu \mathrm{m}$ Spitzer continuum luminosity match those observed by T-ReCS.

Acknowledgements. The authors acknowledge the Spanish MINECO through project Consolider-Ingenio 2010 Program grant CSD2006-00070: First Science with the GTC (http://www.iac.es/consolider-ingenio-gtc/). This work was also partially funded by the Spanish MINECO through a Juan de la Cierva Fellowship. Based on observations obtained at the Gemini Observatory, which is operated by the Association of Universities for Research in Astronomy, Inc., under a cooperative agreement with the NSF on behalf of the Gemini partnership: the National Science Foundation (United States), the Science and Technology Facilities Council (United Kingdom), the National Research Council (Canada), CONICYT (Chile), the Australian Research Council (Australia), Ministerio da Ciencia, Tecnología e Inovacao (Brazil) and Ministerio de Ciencia, Tecnología e Innovación Productiva (Argentina). The Cornell Atlas of Spitzer/IRS Sources (CASSIS) is a product of the Infrared Science Center at Cornell University, supported by NASA and JPL. C. M. Telesco acknowledges support from NSF grant AST-0903672. A.A.-H. and P.E. acknowledge support from the Spanish Plan Nacional de Astronomía y Astrofísica under grant AYA2009-05705-E. A.A.-H. is also supported by the Universidad de Cantabria through the Augusto González Linares Program and by AYA2010-21161-C0201. C.R.A. acknowledges the Estallidos group through project PN AYA201021887-C04.04.

\section{References}

Aalto, S., Johansson, L. E. B., Booth, R. S., \& Black, J. H. 1991, A\&A, 249, 323 Alonso-Herrero, A., Ramos Almeida, C., Mason, R., et al. 2011, ApJ, 736, 82 Alonso-Herrero, A., Sanchez-Portal, M., Ramos Almeida, C., et al. 2012, MNRAS, 425, 311

Antonucci, R. 1993, ARA\&A, 31, 473

Asensio Ramos, A., \& Ramos Almeida, C. 2009, ApJ, 696, 2075

Asmus, D., Gandhi, P., Smette, A., Hönig, S. F., \& Duschl, W. J. 2011, A\&A, 536, A36

Awaki, H., Ueno, S., Koyama, K., Tsuru, T., \& Iwasawa, K. 1996, PASJ, 48, 409 Brandl, B. R., Bernard-Salas, J., Spoon, H. W. W., et al. 2006, ApJ, 653, 1129 Brightman, M., \& Nandra, K. 2011, MNRAS, 413, 1206

Cohen, M., Walker, R. G., Carter, B., et al. 1999, AJ, 117, 1864

Collison, P. M., Saikia, D. J., Pedlar, A., Axon, D. J., \& Unger, S. W. 1994, MNRAS, 268, 203

Comastri, A. 2004, Supermassive Black Holes in the Distant Universe, 308, 245

Davies, R. I., Müller Sánchez, F., Genzel, R., et al. 2007, ApJ, 671, 1388

Dasyra, K. M., Ho, L. C., Netzer, H., et al. 2011, ApJ, 740, 94

De Buizer, J., \& Fisher, R. 2005, High Resolution Infrared Spectroscopy in Astronomy, 84

de La Rosa, I. G., La Barbera, F., Ferreras, I., \& de Carvalho, R. R. 2011, MNRAS, 418, L74

Deo, R. P., Crenshaw, D. M., Kraemer, S. B., et al. 2007, ApJ, 671, 124

Deo, R. P., Richards, G. T., Crenshaw, D. M., \& Kraemer, S. B. 2009, ApJ, 705, 14

de Vaucouleurs, G. 1976, ApJ, 205, 13

Diamond-Stanic, A. M., \& Rieke, G. H. 2010, ApJ, 724, 140

Diamond-Stanic, A. M., \& Rieke, G. H. 2012, ApJ, 746, 168

Díaz-Santos, T., Alonso-Herrero, A., Colina, L., et al. 2008, ApJ, 685, 211

Díaz-Santos, T., Alonso-Herrero, A., Colina, L., et al. 2010, ApJ, 711, 328

Draine, B. T., \& Li, A. 2007, ApJ, 657, 810

Dullemond, C. P., \& van Bemmel, I. M. 2005, A\&A, 436, 47

Ebeling, H., White, D. A., \& Rangarajan, F. V. N. 2006, MNRAS, 368, 65 Elitzur, M. 2008, New Astron. Rev., 52, 274 
Farrah, D., Bernard-Salas, J., Spoon, H. W. W., et al. 2007, ApJ, 667, 149 Fouque, P., Gourgoulhon, E., Chamaraux, P., \& Paturel, G. 1992, A\&AS, 93, 211

Fritz, J., Franceschini, A., \& Hatziminaoglou, E. 2006, MNRAS, 366, 767 Gandhi, P., Horst, H., Smette, A., et al. 2009, A\&A, 502, 457

Garcia, A. M. 1993, A\&AS, 100, 47

Genzel, R., Lutz, D., Sturm, E., et al. 1998, ApJ, 498, 579

Georgantopoulos, I., Dasyra, K. M., Rovilos, E., et al. 2011, A\&A, 531, A116 González Delgado, R. M., Heckman, T., Leitherer, C., et al. 1998, ApJ, 505, 174

González-Martín, O., Masegosa, J., Márquez, I., Guainazzi, M., \& Jiménez-Bailón, E. 2009, A\&A, 506, 1107

Gonzalez-Martin, O., Acosta-Pulido, J. A., Perez Garcia, A. M., \& Ramos Almeida, C. 2010, ApJ, 723, 1748

Goulding, A. D., \& Alexander, D. M. 2009, MNRAS, 398, 1165

Goulding, A. D., Alexander, D. M., Bauer, F. E., et al. 2012, ApJ, 755, 5

Granato, G. L., \& Danese, L. 1994, MNRAS, 268, 235

Granato, G. L., Danese, L., \& Franceschini, A. 1997, ApJ, 486, 147

Guainazzi, M., Rodriguez-Pascual, P., Fabian, A. C., Iwasawa, K., \& Matt, G. 2004, MNRAS, 355, 297

Hao, L., Weedman, D. W., Spoon, H. W. W., et al. 2007, ApJ, 655, L77

Hernán-Caballero, A., Pérez-Fournon, I., Hatziminaoglou, E., et al. 2009, MNRAS, 395, 1695

Higdon, S. J. U., Devost, D., Higdon, J. L., et al. 2004, PASP, 116, 975

Hönig, S. F., \& Kishimoto, M. 2010, A\&A, 523, A27

Hönig, S. F., Beckert, T., Ohnaka, K., \& Weigelt, G. 2006, A\&A, 452, 459

Hönig, S. F., Kishimoto, M., Gandhi, P., et al. 2010, A\&A, 515, A23

Hopkins, P. F. 2012, MNRAS, 420, L8

Horst, H., Gandhi, P., Smette, A., \& Duschl, W. J. 2008, A\&A, 479, 389

Ichikawa, K., Ueda, Y., Terashima, Y., et al. 2012, ApJ, 754, 45

Itoh, T., Done, C., Makishima, K., et al. 2008, PASJ, 60, 251

Jiménez-Bailón, E., Santos-Lleó, M., Dahlem, M., et al. 2005, A\&A, 442, 861

Karachentsev, I., Zonn, W., \& Shcherbanovsky, A. 1972, Astrophys. Lett., 11, 151

Kelm, B., Focardi, P., \& Palumbo, G. G. C. 1998, A\&A, 335, 912

Laine, S., \& Heller, C. H. 1999, MNRAS, 308, 557

Laurent, O., Mirabel, I. F., Charmandaris, V., et al. 2000, A\&A, 359, 887

Lebouteiller, V., Bernard-Salas, J., Sloan, G. C., \& Barry, D. J. 2010, PASP, 122, 231

Lebouteiller, V., Barry, D. J., Spoon, H. W. W., et al. 2011, ApJS, 196, 8

Levenson, N. A., Sirocky, M. M., Hao, L., et al. 2007, ApJ, 654, L45

Levenson, N. A., Radomski, J. T., Packham, C., et al. 2009, ApJ, 703, 390

Maiolino, R., Comastri, A., Gilli, R., et al. 2003, MNRAS, 344, L59

Malkan, M. A., Gorjian, V., \& Tam, R. 1998, ApJS, 117, 25

Marinucci, A., Bianchi, S., Nicastro, F., Matt, G., \& Goulding, A. D. 2012, ApJ, 748,130

Martin, P., Lelièvre, M., \& Roy, J.-R. 2000, ApJ, 538, 141

Mason, R. E., Geballe, T. R., Packham, C., et al. 2006, ApJ, 640, 612

Mason, R. E., Levenson, N. A., Packham, C., et al. 2007, ApJ, 659, 241

Mason, R. E., Lopez-Rodriguez, E., Packham, C., et al. 2012, AJ, 144, 11

Matt, G., Fabian, A. C., Guainazzi, M., et al. 2000, MNRAS, 318, 173

Moran, E. C., Halpern, J. P., \& Helfand, D. J. 1996, ApJS, 106, 341

Mullaney, J. R., Alexander, D. M., Goulding, A. D., \& Hickox, R. C. 2011, MNRAS, 414, 1082

Nenkova, M., Ivezić, Ž., \& Elitzur, M. 2002, ApJ, 570, L9

Nenkova, M., Sirocky, M. M., Ivezić, Ž., \& Elitzur, M. 2008a, ApJ, 685, 147
Nenkova, M., Sirocky, M. M., Nikutta, R., Ivezić, Ž., \& Elitzur, M. 2008b, ApJ, 685,160

Panessa, F., Bassani, L., Cappi, M., et al. 2006, A\&A, 455, 173

Pereira-Santaella, M., Diamond-Stanic, A. M., Alonso-Herrero, A., \& Rieke, G. H. 2010a, ApJ, 725, 2270

Pereira-Santaella, M., Alonso-Herrero, A., Rieke, G. H., et al. 2010b, ApJS, 188, 447

Pereira-Santaella, M., Alonso-Herrero, A., Santos-Lleo, M., et al. 2011, A\&A, 535, A93

Phillips, A. C. 1993 , AJ, 105, 486

Pier, E. A., \& Krolik, J. H. 1992, ApJ, 401, 99

Pier, E. A., \& Krolik, J. H. 1993, ApJ, 418, 673

Ramos Almeida, C., Pérez García, A. M., Acosta-Pulido, J. A., \& Rodríguez Espinosa, J. M. 2007, AJ, 134, 2006

Ramos Almeida, C., Levenson, N. A., Rodríguez Espinosa, J. M., et al. 2009, ApJ, 702, 1127

Ramos Almeida, C., Levenson, N. A., Alonso-Herrero, A., et al. 2011, ApJ, 731, 92

Reduzzi, L., \& Rampazzo, R. 1995, Astrophys. Lett. Comm., 30, 1

Risaliti, G., Elvis, M., Fabbiano, G., et al. 2007, ApJ, 659, L111

Risaliti, G., Miniutti, G., Elvis, M., et al. 2009, ApJ, 696, 160

Roche, P. F., Aitken, D. K., Smith, C. H., \& Ward, M. J. 1991, MNRAS, 248, 606

Roche, P. F., Packham, C., Telesco, C. M., et al. 2006, MNRAS, 367, 1689

Rodriguez Espinosa, J. M., Rudy, R. J., \& Jones, B. 1987, ApJ, 312, 555

Rodriguez Espinosa, J. M., \& Perez-Garcia, A. M. 1997, Astrophys. Lett., 487, 33

Sales, D. A., Pastoriza, M. G., Riffel, R., et al. 2011, ApJ, 738, 109

Schartmann, M., Meisenheimer, K., Camenzind, M., Wolf, S., \& Henning, T. 2005, A\&A, 437, 861

Schartmann, M., Meisenheimer, K., Camenzind, M., et al. 2008, A\&A, 482, 67

Schutte, W. A., Tielens, A. G. G. M., \& Allamandola, L. J. 1993, ApJ, 415, 397

Sengupta, C., \& Balasubramanyam, R. 2006, MNRAS, 369, 360

Shi, Y., Rieke, G. H., Hines, D. C., et al. 2006, ApJ, 653, 127

Siebenmorgen, R., Krügel, E., \& Spoon, H. W. W. 2004, A\&A, 414, 123

Smith, J. D. T., Draine, B. T., Dale, D. A., et al. 2007, ApJ, 656, 770

Spoon, H. W. W., Marshall, J. A., Houck, J. R., et al. 2007, ApJ, 654, L49

Sturm, E., Schweitzer, M., Lutz, D., et al. 2005, ApJ, 629, L21

Sturm, E., Hasinger, G., Lehmann, I., et al. 2006, ApJ, 642, 81

Tacconi-Garman, L. E., Sturm, E., Lehnert, M., et al. 2005, A\&A, 432, 91

Telesco, C. M., Pina, R. K., Hanna, K. T., et al. 1998, Proc. SPIE, 3354, 534

Telesco, C. M., Ciardi, D., French, J., et al. 2003, Proc. SPIE, 4841, 913

Tristram, K. R. W., Meisenheimer, K., Jaffe, W., et al. 2007, A\&A, 474, 837

Tristram, K. R. W., Raban, D., Meisenheimer, K., et al. 2009, A\&A, 502, 67

Trouille, L., Barger, A. J., \& Tremonti, C. 2011, ApJ, 742, 46

Tueller, J., Mushotzky, R. F., Barthelmy, S., et al. 2008, ApJ, 681, 113

Tully, R. B. 1988, AJ, 96, 73

Urry, C. M., \& Padovani, P. 1995, PASP, 107, 803

Veron-Cetty, M.-P., \& Veron, P. 1985, A\&A, 145, 425

Voit, G. M. 1992, MNRAS, 258, 841

Vorontsov-Velyaminov, B. A. 1977, A\&AS, 28, 1

Weedman, D. W., Hao, L., Higdon, S. J. U., et al. 2005, ApJ, 633, 706

Wu, Y., Charmandaris, V., Huang, J., Spinoglio, L., \& Tommasin, S. 2009, ApJ, 701,658 


\section{Appendix A: Catalogue of spectra}
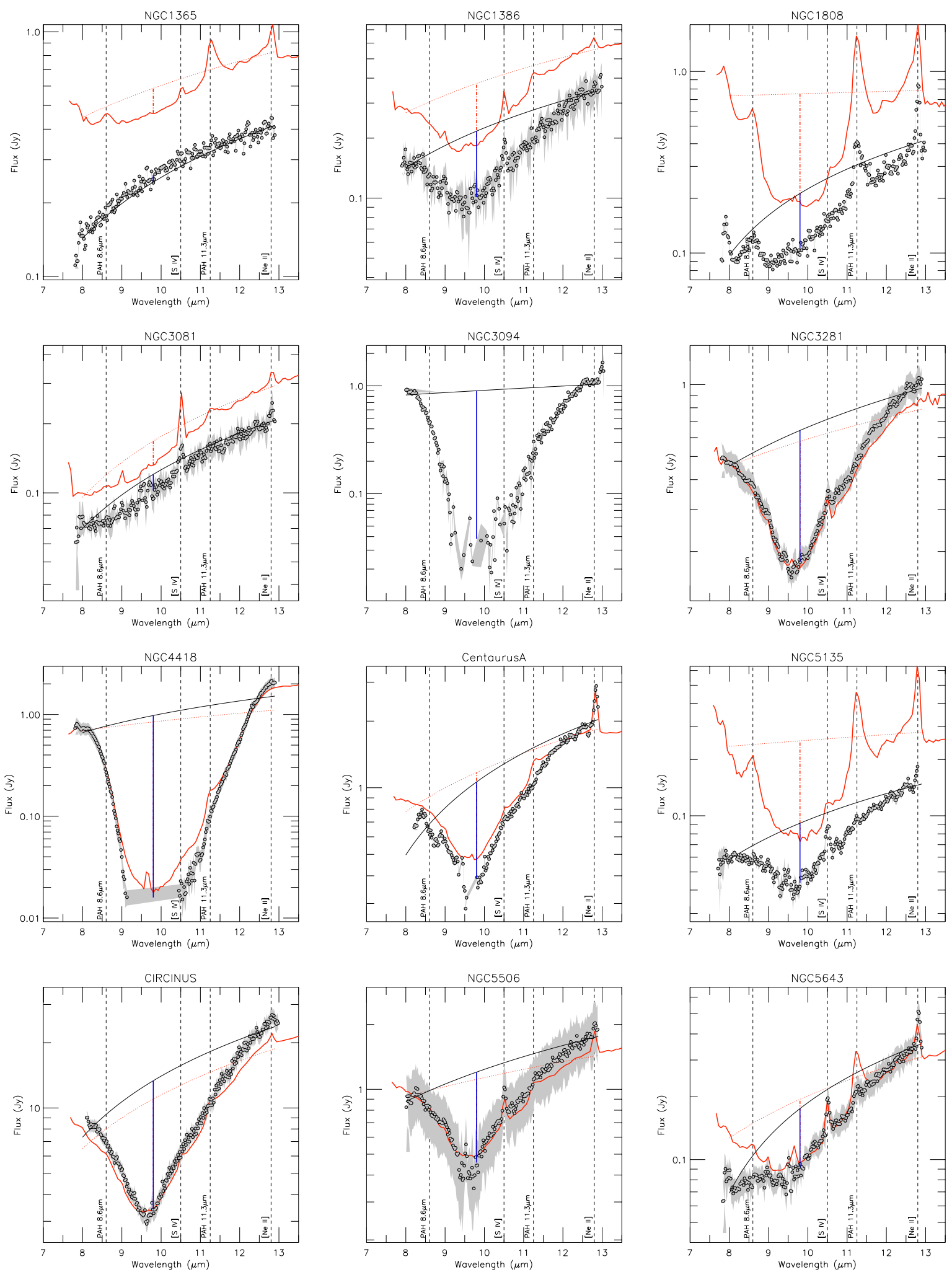

Fig. A.1. T-ReCS spectra of the sample are shown as open circles. The Spitzer/IRS spectra are the red lines. The continuum fits to the T-ReCS and IRS spectra are shown as solid and dotted lines, respectively. Positions of the most prominent emission lines are shown by dashed lines. 
A\&A 553, A35 (2013)
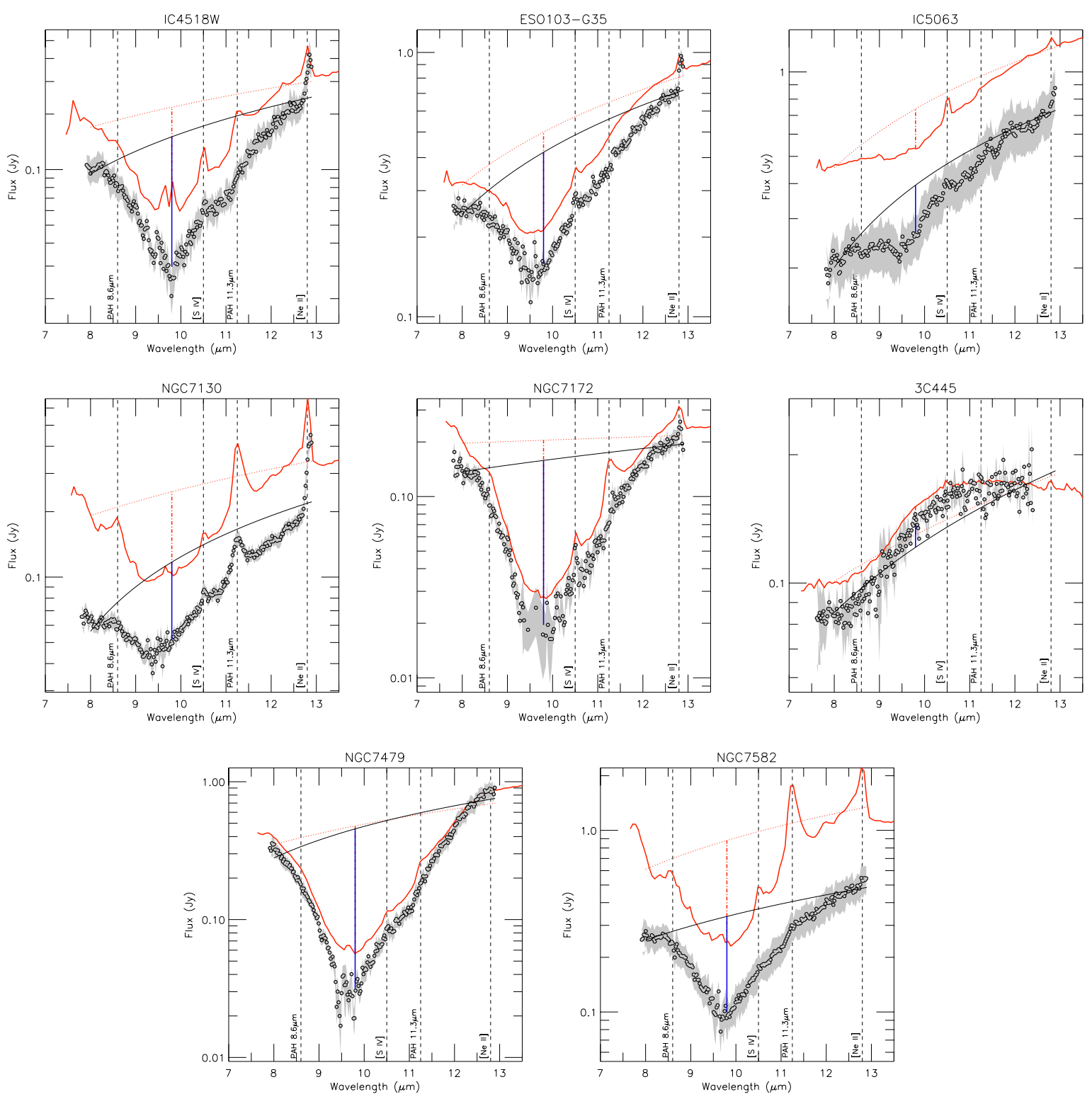

Fig. A.1. continued. 